Pacific Journal of Mathematics

BOUNDS FOR THE EIGENVALUES OF SOME VIBRATING
SYSTEMS 


\section{BOUNDS FOR THE EIGENVALUES OF SOME VIBRATING SYSTEMS}

\section{DALLAS BANKS}

1. Introduction. If a string with a non-negative integrable density $\rho(x), x \in[a, b]$, is fixed at the points $x=a$ and $x=b$ under unit tension, then the natural frequencies of the string are determined by the eigenvalues of the boundary value problem

$$
y^{\prime \prime}+\mu \rho(x) y=0, \quad y(a)=y(b)=0 .
$$

Indicating their dependence on the function $\rho(x)$, we denote these eigenvalues by

$$
\mu_{1}[\rho]<\mu_{2}[\rho]<\cdots .
$$

We consider the set of all such strings which have the same total mass, $M=\int_{a}^{b} \rho(x) d x$. It is well known [5] that the eigenvalues (1.2) satisfy the inequality

$$
\mu_{n}[\rho] \geq \frac{4 n^{2}}{M(b-a)}, \quad n=1,2, \cdots,
$$

with equality when a mass of amount $M / n$ is concentrated at the midpoint of each of $n$ segments obtained by partitioning the string into $n$ equal parts. If we place some restriction on $\rho(x)$ which prohibits such an accumulation of mass, then we can expect to get a larger bound than that of (1.3). M. G. Krein [8] has found that when $0 \leq \rho(x) \leq H<\infty$, the eigenvalues (1.2) satisfy the inequalities

$$
\frac{4 H n^{2}}{M^{2}} X\left(\frac{M}{H(b-a)}\right) \leq \mu_{n}[\rho] \leq \frac{H n^{2} \pi^{2}}{M^{2}},
$$

where $X(t)$ is the least positive root of the equation

$$
\sqrt{X} \tan X=\frac{t}{1-t} .
$$

The inequality (1.4) is sharp and as $H \rightarrow \infty$, the lower bound approaches that of (1.3).

In this paper, we investigate the nature of the density functions for which the greatest lower bounds of the eigenvalues (1.2) are attained

Received June 8, 1959. This research was supported by the United States Air Force Office of Scientific Research.

Submitted in partial fulfillment of the requirements for the degree of Doctor of Philosophy at Carnegie Institute of Technology. 
when other restrictions are placed on $\rho(x)$. For convenience, we may consider the eigenvalue problem

$$
u^{\prime \prime}+\lambda p(x) u=0, \quad u(0)=u(1)=0,
$$

where $p(x)=(b-a) \rho[(b-a) x+a], x \in[0,1]$, instead of (1.1). We note that $\int_{0}^{1} p(x) d x=M$. Denoting the eigenvalues of (1.5) by

$$
\lambda_{1}[p]<\lambda_{2}[p]<\cdots,
$$

we see that

$$
\lambda_{n}[p]=(b-a) \mu_{n}[\rho] .
$$

We shall be concerned with determining bounds for the eigenvalues of the differential system (1.5) under various types of restrictions on $p(x)$. The principal restrictions we shall consider are:

(a) $p(x)$ is monotone in $[0,1]$.

(b) $p(x)$ is convex, i. e., $p(x)$ satisfies the inequality

$$
p(x) \leq \frac{x_{2}-x}{x_{2}-x_{1}} p\left(x_{1}\right)+\frac{x-x_{1}}{x_{2}-x_{1}} p\left(x_{2}\right) .
$$

where $x_{1}$ and $x_{2}$ are any two values such that $0 \leq x_{1} \leq x_{2} \leq 1$.

(c) $p(x)$ is concave, i. e., $-p(x)$ is convex.

These properties are invariant under the linear transformation used to obtain (1.5) from (1.1) so that $\rho(x), x \in[a, b]$, will have the same properties as $p(x)$. Hence, no loss of generality is involved in using the system (1.5).

In $\S 2,3$ and 4 , we obtain sharp lower bounds for $\lambda_{1}[p]$ in these three cases. For the higher eigenvalues we are able to obtain only general information concerning the density distributions which give the lower bounds. The ideas used also lead to results in the case of the more general Sturm-Liouville system

$$
\begin{aligned}
{\left[r(x) u^{\prime}\right]+[\lambda p(x)-q(x)] u } & =0, \\
u^{\prime}(0)-h_{0} u(0)=u^{\prime}(1)+h_{1} u(1) & =0,
\end{aligned}
$$

where $p(x)$ and $q(x)$ are non-negative integrable functions, $r(x) \in C^{\prime}$ is positive and $h_{0} \geq 0, h_{1} \geq 0$. In $\S 5$, we obtain results under various assumptions about $p(x)$ and $q(x)$.

In $\S 6$, we consider the vibrating rod of variable density and with clamped ends. The results we obtain are directly analogous to those obtained by Krein and to those derived in $\S 2,3$ and 4 for the first eigenvalue of (1.5). In $\S 7$, we obtain results for the first eigenvalue of a membrane with fixed boundary in the case of bounded densities and in the case of concave densities on a convex domain. 
The central idea used in finding lower bounds of $\lambda_{1}[p]$ is the following.

Lemma 1.1. If $p(x)$ of (1.5) can be expressed as

$$
p(x)=\int_{0}^{1} K(x, t) g(t) d f(t)
$$

where

(i) $f(t)$ is a monotone increasing bounded function,

(ii) $g(t)$ is non-negative and continuous,

(iii) $K(x, t)$ is non-negative and $\int_{0}^{1} K(x, t) d x=1$,

then

$$
\left.\lambda_{1} \mid p\right] \geq\left[\int_{0}^{1} p(x) d x\right]^{-1} \underset{t \in[0,1]}{\mathrm{g} . \mathrm{l} . \mathrm{b} .} \lambda_{1}[K(x, t)] .
$$

We use the fact that $\lambda_{1}\lfloor p]$ is the minimum of the Rayleigh quotient [4]

$$
J(p, u)=\frac{\int_{0}^{1}\left[u^{\prime}(x)\right]^{2} d x}{\int_{0}^{1} p(x)\lfloor u(x)]^{2} d x}
$$

where $u(x)$ ranges over all functions, with piecewise continuous first derivatives in $[0,1]$, which satisfy the conditions $u(0)=u(1)=0$. In view of (1.8), we have

$$
\lambda_{1}^{-1}[p]=\max _{u} \frac{\int_{0}^{1}\left[\int_{0}^{1} K(x, t) g(t) d f(t)\right] u^{2} d x}{\int_{0}^{1} u^{\prime 2} d x} .
$$

By the properties (i), (ii) and (iii) all terms are non-negative. Interchanging the order of integration, we find that

$$
\lambda_{1}^{-1}\lfloor p] \leq \int_{0}^{1} g(t)\left[\max _{u} \frac{\int_{0}^{1} K(x, t) u^{2} d x}{\int_{0}^{1} u^{\prime 2} d x}\right] d f(t) .
$$

We note that

$$
\lambda_{1}^{-1}[K(x, t)]=\max _{u} \frac{\int_{0}^{1} K(x, t) u^{2} d x}{\int_{0}^{1} u^{\prime 2} d x} .
$$

Hence, (1.11) and (1.12) yield 


$$
\lambda_{1}^{-1}[p] \leq \int_{0}^{1} g(t) d f(t) \operatorname{liu}_{i \in[0,1]} \lambda_{1}^{-1}[K(x, t)] .
$$

From (1.8) and (iii), we have

$$
\int_{0}^{1} p(x) d x=\int_{0}^{1} g(t) d f(t) .
$$

Hence (1.13) is equivalent to (1.9).

If the density $p(x)$ is normalized so that $\int_{0}^{1} p(x) d x=1$, then (1.13) reduces to

$$
\left.\lambda_{1} \mid p\right] \geq \underset{i \in[0,1]}{\operatorname{g.l} . \mathrm{b} .} \lambda_{1}[K(x, t)] .
$$

To obtain results for the higher eigenvalues of (1.5) we use another approach.

LEMMA 1.2. Let $p(x)$ and $q(x)$ be non-negative integrable functions defined for $x \in[a, b]$ and let $f(x)$ be non-negative, continuous and monotone increasing in $[a, b]$. Let $c \in(a, b)$ be such that $p(x) \geq q(x)$ for $x \in[a, c)$ and $p(x) \leq q(x)$ for $x \in(c, b]$. Then

$$
\int_{a}^{b} p(x) d x=\int_{a}^{b} q(x) d x
$$

implies that

$$
\int_{a}^{b} p(x) f(x) d x \leq \int_{a}^{b} q(x) f(x) d x .
$$

If $f(x)$ is monotone decreasing, then the inequality is reversed.

By (1.14) we have

$$
\int_{a}^{c}[q(x)-p(x)] d x=\int_{c}^{b}[p(x)-q(x)] d x .
$$

But $[p(x)-q(x)] \geq 0$ for $x \in[a, c)$ so that the generalized mean-value theorem gives

$$
\int_{a}^{c}[p(x)-q(x)] f(x) d x=f\left(x_{1}\right) \int_{a}^{c}[p(x)-q(x)] d x
$$

for some $x_{1} \in(a, c)$. Similarly, we have

$$
\int_{c}^{b}[q(x)-p(x)] f(x) d x=f\left(x_{2}\right) \int_{c}^{b}[q(x)-p(x)] d x
$$

for some $x_{2} \in(c, b)$. For a monotone increasing $f(x)$, we have $f\left(x_{1}\right) \leq f\left(x_{2}\right)$ so that (1.16), (1.17) and (1.18) imply

$$
\int_{a}^{c}[p(x)-q(x)] f(x) d x \leq \int_{c}^{b}[q(x)-p(x)] f(x) d x .
$$


Adding $\int_{a}^{c} q(x) f(x) d x$ and $\int_{c}^{b} p(x) f(x) d x$ to both sides, we obtain the desired result. If $f(x)$ is monotone decreasing, it is clear that the inequality has to reversed.

M.G. Krein has proved the following result which we will find useful. [8]

Lemma 1.3. Consider a family of density functions $p(x)$ on $[0,1]$ such that $0 \leq p(x) \leq H<\infty$ and $\int_{0}^{1} p(x) d x=M$. Let $\mu=$ g. l. b. $\mu_{1}[p(x)]$ where the greatest lower bound is taken over this family. Then there is a function $p_{0}(x)$ in this family such that $\mu=\lambda_{1}\left\lfloor p_{0}\right]$.

Krein's proof also holds for $\lambda_{n}[p], n=2,3, \cdots$, and for the sum $\sum_{k=1}^{n} \lambda_{k}^{-1}[p]$.

2. Monotone densities. We first consider the system (1.5) when $p(x)$ is a monotone increasing function. We have the following result.

THEOREM 2.1. Let $\lambda_{1}[p]$ be the lowest eigenvalue of a string of unit length with fixed end points whose density is an increasing function $p(x)$. Then

$$
\lambda_{1}[p] \int_{0}^{1} p(x) d x \geq \lambda_{0}
$$

where $\lambda_{0}=7.88 \cdots$. The inequality is sharp and equality is attained for a string whose density is the step function

$$
H\left(x, t_{0}\right)=\left\{\begin{array}{cc}
0, & x \in\left[0, t_{0}\right], \\
\left(1-t_{0}\right)^{-1}, & x \in\left[t_{0}, 1\right],
\end{array}\right.
$$

where $t_{0}=0.357 \cdots$.

Since $p(x)$ is a positive, monotone increasing function, the Stieltjes integral

$$
p(x)-p(0)=\int_{0}^{x} d p(t)
$$

exists for $x \in[0,1]$ except when $\lim _{x \rightarrow 1} p(x)=+\infty$. Even in this case the equality holds in a limiting sense. If we let

$$
h(x, t)= \begin{cases}0, & 0 \leq x \leq t \leq 1 \\ 1, & 0 \leq t \leq x \leq 1\end{cases}
$$

then we have

$$
p(x)=\int_{0}^{1} h(x, t) d p(t)
$$


wherever $p(x)$ is continuous. Here we have replaced the original value of $p(x)$ at $x=0$ by $p(0)=0$; evidently this does not change our result. Since $p(x)$ is monotone, the set of discontinuity points is of zero measure. Hence, for purposes of integration, we may take the above equality to be true everywhere. If we let $H(x, t)=h(x, t)(1-t)^{-1}$, we have

$$
p(x)=\int_{0}^{1} H(x, t)(1-t) d p(t) .
$$

By Lemma 1.1, we then have

$$
\lambda_{1}[p] \geq \underset{i \in[0,1]}{\operatorname{g} .1 . b .} \lambda_{1}[H(x, t)] .
$$

We find the values of $t$ for which the greatest lower bound is attained by solving for $\lambda_{1}[H(x, t)]$ explicitly. If we solve (1.5) in the interval $[0,1]$ with $p(x)$ replaced by $H(x, t)$ we find that over the interval $[t, 1], u(x)$ must satisfy the differential system

$$
u^{\prime \prime}+\frac{\lambda}{1-t} u=0, t u^{\prime}(t)=u(t), u(1)=0 .
$$

The eigenvalues of (2.3) will be equal to $\lambda_{n}[H(x, t)], n=1,2, \ldots$. The eigenfunctions of (2.3) are

$$
u_{n}(x)=\sin z_{n} x+\tan \left[\tan ^{-1} \frac{t z_{n}}{1-t}-\frac{t z_{n}}{1-t}\right] \cos z_{n} t
$$

$n=1,2, \cdots$, where $z_{n}=\sqrt{\lambda_{n}(1-t)}$ is the $n$th positive roots of

$$
\frac{\tan z}{z}=\frac{-t}{1-\mathrm{t}} \text {. }
$$

Hence, the eigenvalues are

$$
\lambda_{n}[H(x, t)]=\frac{z_{n}^{2}}{1-t}, \quad n=1,2, \cdots,
$$

To find the value of $t$ which minimizes $\lambda_{n}[H]=\lambda_{n}[H(x, t)]$ we replace (2.4) by

$$
(1-t) \sin z+t z \cos z=0 \text {. }
$$

This has the same positive zeros as (2.4). Since $\sin z$ and $z \cdot \cos z$ are positive for $0<z<\pi / 2$, (2.6) has no positive zeros in this interval for $t \in[0,1]$. Over the interval $[\pi / 2, \pi)$, $\sin z$ is positive while $z \cdot \cos z$ is negative for $z \in(\pi / 2, \pi]$. Therefore, for $t \in(0,1)$, the left side of (2.6) is positive at $z=\pi / 2$ and negative at $z=\pi$. Hence, (2.6) has its first zero in the interval $[\pi / 2, \pi]$. In fact, only the first one lies in this interval. For if we denote the left side of $(2.6)$ by $F(t, z)$ then 


$$
F_{z}(t, z)=\cos z-t z \sin z
$$

is negative so that for a particular value of $t \in(0,1), F(t, z)$ is monotone decreasing and hence has only one zero for $z \in[\pi / 2, \pi]$. By (2.6)

$$
\frac{d z_{1}}{d t}=\frac{-z_{1}}{1-t+t^{2} z_{1}^{2}}
$$

From (2.5), we have

$$
\frac{d \lambda_{1}[H]}{d t}=\frac{z_{1}}{1-t}\left[2 \frac{d z_{1}}{d t}+\frac{z_{1}}{1-t}\right] .
$$

If we evaluate this at $t=0$, we find $d \lambda_{1} /\left.d t\right|_{t=0}=-\pi^{2}$. Furthermore, since $z_{1}(t)$ is finite, (2.4) implies that $\lambda_{1}[H] \rightarrow+\infty$ as $t \rightarrow 1$, so that $\lambda_{1}[H]$ has a minimum at some $t_{0} \in(0,1)$. Since we are considering only the first zero, $z_{1}(t)$, we will drop the subscript and write $z(t)$. At $t_{0}$ we must have $d \lambda_{1} /\left.d t\right|_{t=t_{0}}=0$ so that (2.8) implies

$$
\left.\frac{d z}{d t}\right|_{t=t_{0}}=-\frac{1}{2} \frac{z\left(t_{0}\right)}{1-t_{0}} .
$$

From (2.7) we find that $z\left(t_{0}\right)=z^{\prime}$ must satisfy

$$
\frac{-z^{\prime}}{1-t_{0}+t_{0}^{2} z^{2}}=-\frac{1}{2} \frac{z^{\prime}}{1-t_{0}} \text {. }
$$

If we solve for $-t_{0}\left(1-t_{0}\right)^{-1}$, it follows from (2.4) that

$$
z^{\prime} \tan z^{\prime}=-t_{0}^{-1} .
$$

Eliminating $t_{0}$ between this and (2.4) we find that $z^{\prime}$ must satisfy

$$
\tan 2 z^{\prime}=2 z^{\prime} .
$$

The first zero of this equation is $z^{\prime} \cong 2.25$. Hence, from (2.4) we find $t_{0}=.357 \cdots$ Now $(2.9)$ has only one zero for $z \in[\pi / 2, \pi]$ so that $\lambda_{1}[H]$ has only one relative extremum for $t \in(0,1)$. But we know there is a minimum so that $t_{0}$ must be the value of $t$ which minimizes $\lambda_{1}[H]$. From (2.5) we find this minimum to be approximately 7.88 .

It does not appear possible to obtain lower bounds for the higher eigenvalues by the exclusive use of Lemma 1.1. We can, however, obtain a bound for the sum $\sum_{k=1}^{n}\left(\lambda_{k}^{-1}[p]\right)$ with the help of a theorem of Courant [5], according to which

$$
\sum_{k=1}^{n} \frac{\int_{0}^{1} p(x) v_{k}^{2} d x}{\int_{0}^{1} v_{k}^{\prime 2} d x}
$$


has the maximum $\sum_{k=1}^{n}\left(\lambda_{k}^{-1}[p]\right)$ if the $v_{k}, k=1,2, \cdots, n$, range over all systems of mutually orthogonal functions with piecewise continuous derivatives in $[0,1]$ such that $v_{k}(0)=v_{k}(1)=0$.

THEOREM 2.2. Let $\lambda_{k}[p], k=1,2, \cdots, n$, be the first $n$ eigenvalues of a string of unit length with fixed ends whose density is an increasing function $p(x)$. If $\lambda_{k}[H(x, t)], k=1,2, \cdots, n$, are the first $n$ eigenvalues of a string whose density is the step function defined by (2.1), then

$$
\left[\int_{0}^{1} p(x) d x\right]^{-1} \sum_{k=1}^{n} \lambda_{k}^{-1}[p] \leq \sum_{k=1}^{n} \lambda_{k}^{-1}\left[H\left(x, t_{0}\right)\right]
$$

where $H\left(x, t_{0}\right)$ is the step function (2.1) and $t_{0}$ is a suitable value in $[0,1]$.

Evidently, the inequality is sharp.

By Courant's theorem, we have for the eigenvalues of the system (1.5)

$$
\sum_{k=1}^{n} \lambda_{k}^{-1}=\max _{v_{k}}\left[\sum_{k=1}^{n} \frac{\int_{0}^{1} p(x) v_{k}^{2} d x}{\int_{0}^{1} v_{k}^{\prime 2} d x}\right],
$$

for suitable $v_{k}$. Using (2.2) and changing the order of integration we have

$$
\sum_{k=1}^{n} \lambda_{k}^{-1}=\max _{v_{k}}\left\{\int_{0}^{1}(1-t)\left[\sum_{k=1}^{n} \frac{\int_{0}^{1} H(x, t) v_{k}^{2} d x}{\int_{0}^{1} v_{k}^{\prime 2} d x}\right] d p(t)\right\} .
$$

Since all the factors are positive we find

$$
\sum_{k=1}^{n} \lambda_{k}^{-1} \leq \int_{0}^{1}(1-t)\left[\max _{v_{k}} \sum_{k=1}^{n} \frac{\int_{0}^{1} H(x, t) v_{k}^{2} d x}{\int_{0}^{1} v_{k}^{\prime 2} d x}\right] d p(t) .
$$

Again by Courant's theorem, we get

$$
\sum_{k=1}^{n} \lambda_{k}^{-1} \leq \int_{0}^{1}(1-t)\left\{\sum _ { k = 1 } ^ { n } \left[\lambda_{k}\left[H(x, t]^{-1}\right\} d p(t)\right.\right.
$$

so that, as in the proof of Lemma 1.1, we have

$$
\sum_{k=1}^{n} \lambda_{k}^{-1} \leq \underset{t \in[0,1]}{1 . u_{0} \text { b. }} \sum_{k=1}^{n}\left[\lambda_{k}[H(x, t)]\right]^{-1} \text {. }
$$

We found in the proof of Theorem 2.1, that $\lambda_{1}[H(x, t)]$ becomes infinite as $t \rightarrow 1$. Hence, $\sum_{k=1}^{n}\left[\lambda_{k}[H(x, t)]\right]^{-1}$ approaches zero as $t \rightarrow 1$. Thus, there is a number $\delta>0$ such that $t \geq 1-\delta$ implies that 


$$
\text { l.u.b. } \sum_{k=1}^{n}\left[\lambda_{k}[H]\right]^{-1} \geq \sum_{k=1}^{n}\left[\lambda_{k}[H(x, t)]\right]^{-1} \text {. }
$$

But for $t \in[0,1-\delta], H(x, t)$ is uniformly bounded. Hence, Lemma 1.3 implies the desired result.

While it seems to be difficult to obtain exact numerical bounds for the higher eigenvalues of the system (1.5), when $p(x)$ is monotone, it is possible to give a geometric characterization of the function $p(x)$ which corresponds to the minimum value of $\lambda_{n}[p]$.

THEOREM 2.3. Let $\lambda_{n}[p]$ be the nth eigenvalue of a string of unit length with fixed ends whose density is a monotone increasing function $p(x)$. Then there is a string with the same total mass whose density is a monotone increasing step function $q(x)$ with at most $n$ jumps such that

$$
\lambda_{n}[p] \geq \lambda_{n}[q],
$$

where $\lambda_{n}[q]$ is the $n$th eigenvalue of the string with density $q(x)$.

Let $u_{n}(x)$ be the $n$th eigenfunction of (1.5) corresponding to $\lambda_{n}[p]$. It is well known that $u_{n}(x)$ has exactly $(n+1)$ zeros in the closed interval $[0,1]$. We denote these zeros by

$$
x_{0}=0<x_{1}<x_{2}<\cdots<x_{n-1}<x_{n}=1 .
$$

In each open subinterval $\left(x_{k}, x_{k+1}\right), u_{n}(x)$ has only one maximum or one minimum so that $u_{n}^{2}(x)$ has a maximum there. We denote these $n$ maximum points by $\bar{x}_{1}<\bar{x}_{2}<\cdots<\bar{x}_{n}$.

We will show presently that it is possible to construct a function $q(x)$ in such a way that over each of the intervals $\left(x_{k-1}, \bar{x}_{k}\right),\left(\bar{x}_{k}, x_{k}\right), k=$ $1,2, \cdots, n, q(x)$ and $p(x)$ are related as indicated in Lemma 1.2. By Lemma 1.2, we will then have

$$
\int_{x_{k-1}}^{\bar{x}_{k}} p(x) u_{n}^{2}(x) d x \leq \int_{x_{k-1}}^{\bar{x}_{k}} q(x) u_{n}^{2}(x) d x, \quad k=1,2, \cdots, n,
$$

and

$$
\int_{\bar{x}_{k}}^{x_{k}} p(x) u_{n}^{2}(x) d x \leq \int_{\bar{x}_{k}}^{x_{k}} q(x) u_{n}^{2}(x) d x, \quad k=1,2, \cdots, n
$$

Upon adding these inequalities, we get

$$
\int_{x_{k-1}}^{x_{k}} p(x) u_{n}^{2}(x) d x \leq \int_{x_{k-1}}^{x_{k}} q(x) u_{n}^{2}(x) d x, \quad k=1,2, \cdots, n .
$$

If we fix the string at the nodal points $x_{k}, k=0,2, \cdots, n$, then it is known [5] that 


$$
\lambda_{n}=\frac{\int_{x_{k-1}}^{x_{k}} u_{n}^{\prime 2} d x}{\int_{x_{k-1}}^{x_{k}} p(x) u_{n}^{2} d x}, \quad k=1,2, \cdots, n
$$

By (2.11), we have

$$
\lambda_{n} \geq \frac{\int_{x_{k-1}}^{x_{k}} u_{n}^{\prime 2} d x}{\int_{x_{k-1}}^{x_{k}} q(x) u_{n}^{2} d x} \geq \min _{u \in O^{\prime}} \frac{\int_{x_{k-1}}^{x_{k}} u^{\prime 2} d x}{\int_{x_{k-1}}^{x_{k}} q(x) u^{2} d x}
$$

where $u\left(x_{k}\right)=0, k=1,2, \cdots, n$. In particular, $\lambda_{n}$ must satisfy

$$
\lambda_{n} \geq \max _{1 \leq k \leq n}\left[\min _{u \in O^{\prime}} \frac{\int_{x_{k-1}}^{x_{k}} u^{\prime 2} d x}{\int_{x_{k-1}}^{x_{k}} q(x) u^{2} d x}\right] .
$$

But the quantity on the right is greater than the $n$th eigenvalue $\lambda_{n}[q]$ of a string with density $q(x)$ so (2.10) will hold (See [5]).

It remains to be shown that there exists a function $q(x)$ of the desired form.

We first consider the intervals $\left(\bar{x}_{k}, x_{k}\right], k=1,2, \cdots, n$. Here we set

$$
q(x)=\left(x_{k}-\bar{x}_{k}\right)^{-1} \int_{\bar{x}_{k}}^{x_{k}} p(x) d x=a_{k}, \quad k=1,2, \cdots, n .
$$

Since $p(x)$ is monotone increasing, the hypothesis of lemma (1.2) is obviously satisfied. For the intervals $\left(x_{k-1}, \bar{x}_{k}\right)$, we choose a point $t_{k} \in$ $\left[x_{k-1}, \bar{x}_{k}\right]$ such that

$$
\left(\bar{x}_{k}-t_{k}\right)\left(a_{k}-a_{k-1}\right)=\int_{x_{k-1}}^{\bar{x}_{k}} p(x) d x-a_{k-1}\left(\bar{x}_{k}-x_{k-1}\right)
$$

$k=1,2, \cdots, n$, where we take $a_{0}=0$ and set

$$
q(x)=\left\{\begin{array}{l}
a_{k-1}, x \in\left(x_{k-1}, t_{k}\right], \\
a_{k}, x \in\left(t_{k}, \bar{x}_{k}\right],
\end{array}\right.
$$

$k=1,2, \cdots, n$. By the definition of the $a_{k}$ 's, $p(x) \geq a_{k-1}, x \in\left[x_{k-1}, t_{k}\right)$ and $p(x) \leq a_{k}$ for $x \in\left[t_{k}, \bar{x}_{k}\right)$ so that the hypothesis of Lemma 1.2 is again satisfied. Thus, the function $q(x), x \in[0,1]$ may be taken to be

$$
q(x)=a_{k}, x \in\left(t_{k}, t_{k+1}\right), \quad k=0,1,2, \cdots, n,
$$

where we let $t_{0}=0$ and $t_{n+1}=1$. This proves the theorem.

3. Convex densities. We now turn to the consideration of (1.5) in the case where $p(x)$ is convex. We have the following theorem. 
THEOREM 3.1. Let $\lambda_{1}\lfloor p\rfloor$ be the first eigenvalue of a string of unit length with fixed ends whose density is a continuous convex function $p(x)$. Then

$$
\lambda_{1}[p] \int_{0}^{1} p(x) d x \geq \lambda_{0}
$$

where $\lambda_{0}=9.397 \ldots$. The inequality is sharp and equality is attained for a string whose density is the piecewise linear function

$$
G\left(x, t_{0}\right)= \begin{cases}0 & , \quad x \in\left[0, t_{0}\right], \\ 2 \frac{x-t_{0}}{\left(1-t_{0}\right)^{2}}, & x \in\left[t_{0}, 1\right],\end{cases}
$$

where $t_{0}=0.104 \cdots$.

We first note that any positive convex density $p(x)$ may be written as the sum $p_{1}(x)+p_{2}(x)$ where $p_{1}(x)$ is a positive monotone increasing convex function and $p_{2}(x)$ is a positive monotone decreasing convex function. In particular, we may define $p_{1}(x)$ such that $p_{1}(0)=0$ and $p_{2}(x)$ such that $p_{2}(1)=0$. If $\xi \in[0,1]$ is a minimum point of $p(x)$, it is easily confirmed that the functions

$$
p_{1}(x)= \begin{cases}p(\xi) x & , x \in[0, \xi], \\ p(x)-p(\xi)(1-x) & , x \in[\xi, 1]\end{cases}
$$

and

$$
p_{2}(x)= \begin{cases}p(x)-p(\xi) x, & x \in[0, \xi], \\ p(\xi)(1-x), & x \in[\xi, 1],\end{cases}
$$

have the required properties.

We may thus express $p(x)$ as $p(x)=\alpha \bar{p}_{1}(x)+\beta \bar{p}_{2}(x)$, where $M \alpha=$ $\int_{0}^{1} p_{1}(x) d x, M \beta=\int_{0}^{1} p_{2}(x) d x, \bar{p}_{1}(x)=p_{1}(x) / \alpha$ and $\bar{p}_{2}(x)=p_{2}(x) / \beta$. From the Rayleigh quotient we then have

$$
\begin{aligned}
\lambda_{1}^{-1}[p] & =\max _{u \in O^{\prime}}[J(p, u)]^{-1} \\
& \leq \max _{u \in O^{\prime}} \alpha\left[J\left(\bar{p}_{1}, u\right)\right]^{-1}+\max _{n \in O^{\prime}} \beta\left[J\left(\bar{p}_{2} u\right)\right]^{-1} .
\end{aligned}
$$

Let $\lambda_{1}\left[\bar{p}_{1}\right]$ and $\lambda_{1}\left[\bar{p}_{2}\right]$ be the first eigenvalues of strings with fixed end points and densities $\bar{p}_{1}(x)$ and $\bar{p}_{2}(x)(x \in[0,1])$ respectively. Then, from (3.2),

$$
\lambda_{1}[p] \geq \min _{(1,2)}\left(\lambda_{1}\left[\bar{p}_{1}\right], \lambda_{1}[\bar{p}]\right) .
$$

Because of the symmetry of the boundary conditions $u(0)=u(1)=0$, it is obvious that the bound for $\lambda_{1}[p]$ in the case of monotone increasing 
$p(x)$ is the same as that for monotone decreasing $p(x)$. Hence, we need only consider monotone increasing functions. Furthermore, as shown above, we may assume that $p(0)=0$.

Now set

$$
g(x, t)= \begin{cases}0, & x \in[0, t], \\ x-t, & x \in[t, 1],\end{cases}
$$

where $t \in[0,1]$. We first assume that the increasing function $p(x)$ is bounded and that the left-hand derivative $p_{-}^{\prime}(x)$ is bounded for $x \in[0,1]$. It then follows from integration by parts and the fact that

$$
\int_{0}^{x} p_{-}^{\prime}(t) d t=p(x)-p(0),
$$

for such a function $p(x)[13]$, that

$$
p(x)=p_{-}^{\prime}(0) x+\int_{0}^{1} g(x, t) d p_{-}^{\prime}(t) .
$$

If we set $G(x, t)=\left(2 /(1-t)^{2}\right) g(x, t)$, we have

$$
p(x)=\int_{0}^{1} G(x, t)\left[1 / 2(1-t)^{2}\right] d p_{-}^{\prime}(t) .
$$

Here we have replaced the original value of $p_{-}^{\prime}(x)$ at $x=0$, by $p_{-}^{\prime}(0)=0$; evidently this does not change our result. By Lemma 1.1, we then have

$$
\lambda_{1}[p] \geq \underset{t \in[0.1]}{\operatorname{g.j} . \text { b. }} \lambda_{1}[G(x, t)]\left[\int_{0}^{1} p(x) d x\right]^{-1} .
$$

If $p(x)$ or its left derivative is not bounded in $[0,1]$, we may consider the system

$$
u^{\prime \prime}+\lambda p(x) u=0, u(0)=u(1-\varepsilon)=0
$$

where $\varepsilon>0$ is arbitrarily small. In the interval $[0,1-\varepsilon], p(x)$ and $p_{-}^{\prime}(x)$ are bounded, so transforming the system (3.4) to the unit interval we find, by (3.3), that

$$
(1-\varepsilon) \lambda_{1}[p]_{\varepsilon} \int_{0}^{1-\varepsilon} p(x) d x \geq g_{t \in[0,1]} \text { l. b. }_{0} \lambda_{1}[G(x, t)],
$$

where $\lambda_{1}[p]_{\varepsilon}$ is the first eigenvalue of (3.4). Since $\varepsilon$ is arbitrary and the eigenvalues are continuous functions of the length of the interval, it follows that (3.3) holds for any increasing convex density $p(x)$.

To find the values of $t$ for which the greatest lower bound of $\lambda_{1}[p]$ is attained, we employ a procedure similar to that used in a corresponding situation in the proof of Theorem 2.1. Our problem is then seen to reduce to the computation of the lowest eigenvalue of the system 


$$
u^{\prime \prime}(x)+\frac{2 \lambda}{(1-t)^{2}}(x-t) u(x)=0, t v^{\prime}(t)=v(t), v(1)=0 .
$$

The eigenfnnctions of (3.5) are [7]

$$
u_{n}(x)=\sqrt{x-t}\left[J_{1 / 3}\left(z_{n}\left(\frac{x-t}{1-t}\right)^{3 / 2}\right)+\frac{t \Gamma(2 / 3)\left(6 \lambda_{n}\right)^{1 / 3}}{(1-t)^{2 / 3} \Gamma(1 / 3)} J_{-1 / 3}\left(z_{n}\left(\frac{x-t}{1-t}\right)^{3 / 2}\right)\right]
$$

where $J_{ \pm(1 / 3)}(y)$ are Bessel functions of order $\pm 1 / 3$ and $z_{n}=(2 / 3) \sqrt{2 \lambda}(1-t)^{-1}$ is the $n$th positive root of

$$
(1-t) \Gamma\left(\frac{4}{3}\right)\left(\frac{z}{2}\right)^{-1 / 3} J_{1 / 3}(z)+t \Gamma\left(\frac{2}{3}\right)\left(\frac{z}{2}\right)^{1 / 3} J_{-1 / 3}(z)=0 .
$$

Hence, the eigenvalues of (3.5) are

$$
\lambda_{n}[G(x, t)]=\frac{9 z_{n}^{2}}{8(1-t)}, \quad n=1,2, \cdots .
$$

We denote the left side of (3.6) by $F(t, z)$. To find the value $t_{0}$ which minimizes $\lambda_{1}[G]$, we must investigate some properties of this function. The first positive zeros of $J_{1 / 3}(z)$ and $J_{-1 / 3}(z)$ are $\xi_{0}=1.87$ and $\xi_{1}=2.90$, respectively [9]. In $\left(0, \xi_{0}\right), F(0, z)$ and $F(1, z)$ are positive. Hence, $F(t, z)$ has no zeros in this interval for $t \in(0,1)$. In $\left[\xi_{0}, \xi_{1}\right), F(0, z)$ is positive, while $F(1, z)$ is negative in $\left(\xi_{0}, \xi_{1}\right]$. Accordingly, for $t \in(0,1), F\left(t, \xi_{0}\right)$ is negative while $F\left(t, \xi_{1}\right)$ is positive. Hence, $F(t, z)$ has its first zero in $\left(\xi_{0}, \xi_{1}\right)$. Furthermore, there is only one zero in this interval for a given value of $t$, since

$$
-F_{z}(t, z)=(1-t) \Gamma(4 / 3)(z / 2)^{-1 / 3} J_{4 / 3}(z)+t \Gamma(2 / 3)(z / 2)^{1 / 3} J_{2 / 3}(z),
$$

and it is known that each of the terms on the right side is positive for $z \in\left(\xi_{0}, \xi_{1}\right)$. Thus, for a given $t, F(t, z)$ is monotone decreasing over this interval and thus has only one zero there.

Since we are considering only the first zero $z_{1}(t)$, we will drop the subscript and write $z(t)$. We have

$$
\frac{d z}{d t}=\frac{\Gamma(2 / 3)(z / 2)^{1 / 3} J_{-1 / 3}(z)-\Gamma(4 / 3)(z / 2)^{-1 / 3} J_{1 / 3}(z)}{(1-t) \Gamma(4 / 3)(z / 2)^{-1 / 3} J_{4 / 3}(z)+t \Gamma(2 / 3)(z / 2)^{1 / 3} J_{2 / 3}(z)},
$$

and by (3.7),

$$
\frac{d \lambda_{1}[G]}{d t}=\frac{9 z}{8(1-t)}\left[2 \frac{d z}{d t}+\frac{z}{1-t}\right]
$$

If we evaluate this at $t=0$, we find $\left(d \lambda_{1}[G]\right) /\left.d t\right|_{t=0}=-1.38$. Furthermore, since $z(t)$ is finite for all $t \in[0,1],(3.7)$ implies that for $t \rightarrow 1$, $\lambda_{1}[G] \rightarrow+\infty$ so that $\lambda_{1}[G]$ has a minimum at some value $t_{0} \in(0,1)$. At an 
extremum $t^{\prime}$ of $\lambda_{1}[G]$ we must have $\left(d \lambda_{1}[G]\right) /\left.d t\right|_{t^{\prime}}=0$, so that (3.9) implies

$$
\left.\frac{d z}{d t}\right|_{t=t^{\prime}}=-\frac{1}{2} \frac{z\left(t^{\prime}\right)}{1-t^{\prime}}
$$

If we substitute (3.8) into (3.10) and then eliminate $t^{\prime}$ between this result and (3.5), we get

$$
\begin{gathered}
\Gamma(4 / 3)\left(z^{\prime} / 2\right)^{2 / 3} J_{1 / 3}\left(z^{\prime}\right) J_{2 / 3}\left(z^{\prime}\right)=\Gamma(2 / 3)\left(z^{\prime} / 2\right)^{1 / 3}\left[J_{1 / 3}\left(z^{\prime}\right)\right]^{2} \\
\left.-\Gamma(4 / 3)\left(z^{\prime} / 2\right)^{-1 / 3} J_{1 / 3}\left(z^{\prime}\right) J_{-1 / 3}\left(z^{\prime}\right)-\left(z^{\prime} / 2\right)^{2 / 3} J_{4 / 3}\left(z^{\prime}\right) J_{-1 / 3}\left(z^{\prime}\right)\right],
\end{gathered}
$$

where $z^{\prime}=z\left(t^{\prime}\right)$. If we use the relations [7],

$$
\begin{gathered}
J_{4 / 3}(x)=(1 / 3)(x / 2)^{-1} J_{1 / 3}(x)-J_{-2 / 3}(x), \\
J_{1 / 3}(x) J_{2 / 3}(x)+J_{-1 / 3}(x) J_{-2 / 3}(x)=\frac{2 \sin \pi / 3}{\pi x}
\end{gathered}
$$

and

$$
\Gamma\left(\frac{2}{3}\right) \Gamma\left(\frac{1}{3}\right)=\pi / \sin \pi / 3
$$

we finally arrive at the equation

$$
\begin{aligned}
& {\left[\Gamma\left(\frac{2}{3}\right)\left(\frac{z^{\prime}}{2}\right)^{1 / 3} J_{-1 / 3}\left(z^{\prime}\right)\right]\left[\Gamma\left(\frac{2}{3}\right)\left(\frac{z^{\prime}}{2}\right)^{1 / 3} J_{-1 / 3}\left(z^{\prime}\right)\right.} \\
&\left.-\frac{2}{3} \Gamma\left(\frac{4}{3}\right)\left(\frac{z^{\prime}}{2}\right)^{-1 / 3} J_{1 / 3}\left(z^{\prime}\right)\right]-\frac{1}{3}=0 .
\end{aligned}
$$

We show that this equation has only one zero in $\left(\xi_{0}, \xi_{1}\right)$, i.e., that $\lambda_{1}[G]$ has only one extremum for $t \in(0,1)$. This will be the case if the derivative of the expression on the left of (3.11) is of one sign for $z^{\prime} \in\left(\xi_{0}, \xi_{1}\right)$. The following statements relate to this interval.

(i) $\left[\Gamma(2 / 3)\left(z^{\prime} / 2\right)^{1 / 3} J_{-1 / 3}\left(z^{\prime}\right)\right]$, is negative and decreasing.

(ii) $-(2 / 3) \Gamma(4 / 3)\left(z^{\prime} / 2\right)^{-1 / 3} J_{1 / 3}\left(z^{\prime}\right)$, is negative so that the quantity in the second bracket also is negative.

(iii) The second bracket is decreasing.

The last statement requires verification. The derivative of the quantity in question is

$$
-\left[\Gamma\left(\frac{2}{3}\right)\left(\frac{z^{\prime}}{2}\right)^{1 / 3} J_{2 / 3}\left(z^{\prime}\right)-(2 / 3) \Gamma\left(\frac{4}{3}\right)\left(\frac{z^{\prime}}{2}\right)^{-1 / 3} J_{4 / 3}\left(z^{\prime}\right)\right] .
$$

(iii) will be verified if we show that

$$
\frac{3 \Gamma(2 / 3)}{2 \Gamma(4 / 3)}\left(z^{\prime} / 2\right)^{2 / 3} J_{2 / 3}\left(z^{\prime}\right)=J_{4 / 3}\left(z^{\prime}\right)>0,
$$


for $z \in\left(\xi_{0}, \xi_{1}\right)$. Since

$$
\frac{d}{d z}(z / 2)^{2 / 3} J_{2 / 3}(z)=(z / 2)^{2 / 3} J_{-1 / 3}(z)<0,
$$

we have

$$
\left(z^{\prime} \mid 2\right)^{2 / 3} J_{2 / 3}\left(z^{\prime}\right) \geq\left(\xi_{1} / 2\right)^{2 / 3} J_{2 / 3}\left(\xi_{1}\right) \text {. }
$$

Furthermore $\max _{\xi_{0} \leq z \leq \xi_{1}} J_{4 / 3}(z)<.52$. Evaluation of (3.12) then gives the desired result.

We thus conclude that the left-hand side of (3.11) is increasing and hence, that $\lambda_{1}[G]$ has only one extremum for $t \in(0,1)$. But we know that there is at least one minimum so that it must be determined by (3.11), i.e., $t_{0}=t^{\prime}$. From (3.11) we find by Newton's method that $z_{1}\left(t_{0}\right)=2.73$ so that $t_{0}=.104 \cdots$. Therefore, we find that

$$
\left.\left.\lambda_{1}[G(x, t)] \geq \lambda_{1}\right] G\left(x, t_{0}\right)\right]=9.397 \cdots .
$$

Corresponding to Theorem 2.2, we have the following.

THEOREM 3.2. Let $\lambda_{k}[p], k=1,2, \cdots, n$ be the first $n$ eigenvalues of a string of unit length with fixed ends whose density is a continuous convex function $p(x)$. If $\lambda_{k}[G(x, t)], k=1,2, \cdots, n$ are the first $n$ eigenvalues of a string whose density is the convex increasing function $G(x, t)$ defined in (3.1), then

$$
\left[\int_{0}^{1} p(x) d x\right]^{-1} \sum_{k=1}^{n} \lambda_{k}^{-1}[p] \leq \sum_{k=1}^{n} \lambda_{k}^{-1}\left[G\left(x, t_{0}\right)\right]
$$

where $t_{0}$ is a suitable value in $[0,1]$.

Evidently, the inequality is sharp. The proof has the same forma? relationship to that of Theorem 3.1 as the proof of Theorem 2.2 had tc that of Theorem 2.1. Since no additional ideas are involved, we omit the proof.

Theorem 3.1 can be used to obtain an explicit lower bound for the second eigenvalue $\lambda_{2}[p]$ of the system (1.5) when $p(x)$ is convex.

THEOREM 3.3. Let $\lambda_{2}[p]$ be the second eigenvalue of a string with convex density. Then

$$
\lambda_{2}[p] \int_{0}^{1} p(x) d x \geq 4 \lambda_{0},
$$

where $\lambda_{0}$ is the value defined in Theorem 3.1. This inequality is sharx and equality is attained for a string with density $q(x)$ where

$$
q(x)=\left\{\begin{array}{l}
G\left(1-2 x, t_{0}\right), x \in[0,1 / 2], \\
G\left(2 x-1, t_{0}\right), x \in[1 / 2,1],
\end{array}\right.
$$


$G(x, t)$ and $t_{0}$ being defined as in Theorem 3.1.

Let $u_{2}(x)$ be the eigenfunction corresponding to $\lambda_{2}[p]$. Then $u_{2}(x)$ has exactly one nodal point $x_{1} \in(0,1)$. If we hold the string fixed at this point, we get two independent strings, each of which has the lowest eigenvalue $\lambda_{2}[p]$. By Theorem 3.1 and equation (1.6), the lowest eigenvalue of a string with a convex density satisfies the inequalities

$$
\lambda_{2}[p] \geq \frac{\lambda_{0}}{x_{1} \int_{0}^{x_{1}} p(x) d x}=\mu^{\prime}
$$

and

$$
\lambda_{2}[p] \geq \frac{\lambda_{0}}{\left(1-x_{1}\right) \int_{x_{1}}^{1} p(x) d x}=\mu^{\prime \prime} .
$$

We may take the density function for which the bound in (3.13) is attained to be

$$
\bar{p}(x)=\alpha G\left(\frac{x_{1}-x}{x_{1}}, t_{0}\right), x \in\left[0, x_{1}\right],
$$

where $\alpha$ is such that $\int_{0}^{x} \bar{p}(x) d x=\int_{0}^{x_{1}} p(x) d x$. For the second segment the bound is attained for the density function

$$
\bar{p}(x)=\beta G\left(\frac{x-x_{1}}{1-x_{1}}, t_{0}\right), x \in\left[x_{1}, 1\right]
$$

where $\beta$ is such that $\int_{x_{1}}^{1} \bar{p}(x) d x=\int_{0}^{x_{1}} p(x) d x$.

Now, consider a string whose density function is defined by piecing together the above densities at $x_{1}$. This particular choice of $\bar{p}(x)$ then gives us a convex function. The second eigenvalue $\lambda_{2}[\bar{p}]$, of the resulting string satisfies the relationship $\lambda_{2}[\bar{p}] \leq \max \left(\mu^{\prime}, \mu^{\prime \prime}\right)$. Hence, (3.13) and (3.14) imply that

$$
\lambda_{2}[p] \geq \lambda_{2}[\bar{p}],
$$

where $\lambda_{2}[\bar{p}]$ is the second eigenvalue of

$$
u^{\prime \prime}+\lambda \bar{p}(x) u=0, u(0)=u(1)=0 .
$$

We now consider this system with $\bar{p}(x)$ replaced by

$$
\bar{q}(x)=\left\{\begin{array}{l}
\alpha G\left(\frac{x_{1}-x}{x}, t_{0}\right) x \in\left[0, x_{1}\right], \\
\beta G\left(\frac{x_{1}-x}{1-x_{1}}, t_{0}\right) x \in\left[x_{1}, 1\right],
\end{array}\right.
$$


where $\alpha$ and $\beta$ are now determined by the conditions $\int_{0}^{1} \bar{q}(x) d x=M, \alpha \geq$ $0, \beta \geq 0$ and where $x_{1} \in[0,1]$ is a free parameter. It is clear that g.l. $b_{{ }_{\left(\alpha, \beta, x_{1}\right)}}$ $\lambda_{2}[\bar{q}] \leq \lambda_{2}[\bar{p}] \leq \lambda_{2}[p]$. We may let $\theta M=\int_{0}^{x_{1}} \bar{q}(x) d x$ so that $\int_{x_{1}}^{1} \bar{q}(x) d x=$ $(1-\theta) M$. We now show that smallest possible value of $\lambda_{2}[\bar{q}]$ is attained when $x_{1}=1 / 2$ and $\theta=1 / 2$.

Let $\lambda_{2}[\bar{q}]=\lambda_{2}\left(x_{1}, \theta\right)$. We first show that $M \lambda_{2}\left(x_{1}, \theta\right)>4 \lambda_{0}$ if $x_{1} \in[0,1 / 4)$ or $x_{1} \in(3 / 4,1]$. Assume $x_{1} \in[0,1 / 4)$ and consider the case where $x_{1}^{\prime}$, the nodal point of the eigenfunction corresponding to $\lambda_{2}\left(x_{1}, \theta\right)$, lies in the interval $[0,1 / 4)$. If we hold the string fixed at $x_{1}^{\prime}$, the resulting segments each have a lowest eigenvalue $\lambda_{2}\left(x_{1}, \theta\right)$. In particular, $\lambda_{2}\left(x_{1}, \theta\right)$ is the lowest eigenvalue of the segment $\left[0, x_{1}^{\prime}\right]$. By Theorem 3.1 and equation (1.6), we have

$$
\lambda_{2}\left(x_{1} \theta\right) \geq \frac{\lambda_{0}}{x_{1}^{\prime} \int_{0}^{x_{1}^{\prime}} \bar{q}(x) d x} \geq \frac{\lambda_{0}}{x^{\prime} M} .
$$

But $x_{1}^{\prime}<1 / 4$ so that $M \lambda_{2}\left(x_{1}, \theta\right)>4 \lambda_{0}$. It follows in the same way that if $x_{1}^{\prime} \in(3 / 4,1]$ then $\lambda_{2}\left(x_{1}, \theta\right) \geq \lambda_{0}\left(1-x_{1}^{\prime}\right)^{-1} \cdot M^{-1}$ and hence we again have $M \lambda_{2}\left(x_{1}, \theta\right)>4 \lambda_{0}$. Similarly if $x_{1} \in(3 / 4,1]$ we conclude that $M \lambda_{2}\left(x_{1}, \theta\right)>4 \lambda_{0}$ so that this inequality holds unless $x_{1} \in[1 / 4,3 / 4]$.

Hence, we consider the system (3.16) with $\bar{q}(x)$ in place of $\bar{p}(x)$ where $x_{1} \in[1 / 4,3 / 4]$. With $x_{1}$ restricted in this manner, it follows that the family of density functions $\bar{q}(x)$ is bounded uniformly. Hence, by Lemma 1.3 , there are value $x_{1}^{0}$ and $\theta^{0}$ such that $\lambda_{2}\left(x_{1}^{0}, \theta^{0}\right)=\min _{x_{1}, \theta} \lambda_{2}\left[x_{1}, \theta\right]$ for some density $\bar{q}^{0}(x)$.

We first note that $x_{1}^{0}$ must be a nodal point of the corresponding eigenfunction, for otherwise we could hold the string fixed at the nodal point and find another density which would give a lower second eigenvalue by the process which was used to obtain (3.15).

Thus, the lowest second eigenvalue is given by

$$
M \lambda_{2}\left[x_{1}^{0}, \theta^{0}\right]=\frac{\lambda_{0}}{x_{1}^{0} \theta^{0}}=\frac{\lambda_{0}}{\left(1-x_{1}^{0}\right)\left(1-\theta^{0}\right)} .
$$

Solving for $\theta^{0}$, we find $\theta^{0}=1-x_{1}^{0}$, so that $\lambda_{2}\left[x_{1}^{0}, \theta^{0}\right]=\lambda_{0} /\left(x_{1}^{0}\left(1-x_{1}^{0}\right)\right)$. This is obviously smallest when $x_{1}^{0}=1 / 2$, so that $\theta^{0}=1 / 2$. By (1.15) we then have the desired result.

We now consider the higher eigenvalues of the system (1.5) when $p(x)$ is convex. Unfortunately, we cannot use the technique just described for $\lambda_{2}[p]$, since the resulting function will, in general, not be convex. It is, however, possible to obtain a geometric characterization of the extremal density. 
THEOREM 3.4. Let $\lambda_{n}[p]$ be the $n$th eigenvalue of a string of unit length with fixed ends whose density is a continuous convex function $p(x)$. Then there is a string with the same total mass whose density is a piecewise linear convex function $q(x)$ with at most $n+1$ distinct linear segments such that

$$
\lambda_{n}[p] \geq \lambda_{n}[q]
$$

where $\lambda_{n}[q]$ is the $n$th eigenvalue of the string with density $q(x)$.

Let $u_{n}(x)$ be the $n$th eigenfunction of (1.5) when $p(x)$ is convex. As in the proof of Theorem 2.3, we use the fact that $\lambda_{n}=J\left(p, u_{n}\right)$, where $J(p, u)$ is the Rayleigh quotient (1.10). If we construct a function $q(x)$ such that the inequality (2.11) is satisfied, then it follows as in the proof of Theorem 2.3 that $\lambda_{n}[q] \leq \lambda_{n}[p]$. Hence, it remains to be shown that such a function $q(x)$ exists.

We begin by carrying out a preliminary construction. As in Theorem 2.3, we denote the minimum points of $u_{n}^{2}(x)$ by $x_{k}, k=0,1, \cdots, n$, and the maximum points by $\bar{x}_{k}, k=1,2, \cdots, n$. We consider each of the intervals $\left(x_{k-1}, x_{k}\right) k=1,2, \cdots, n$ separately. Let $a(x)$ be any linear function such that $a(x) \leq p(x), x \in\left[x_{k-1}, x_{k}\right]$. Then $r(x)=\max [a(x), 0]$ satisfies the inequality $0 \leq r(x) \leq p(x)$.

We now consider one of the intervals, say $\left(x_{k-1}, x_{k}\right)$, where $1 \leq k \leq n$. Let $c_{k}$ be any' number such that $c_{k} \geq p\left(\bar{x}_{k}\right)$. Then there is a number $a_{k}$ such that

$$
\int_{x_{k-1}}^{\bar{x}_{k}}\left[a_{k}\left(x-x_{k}\right)+c_{k}\right] d x=\int_{x_{k-1}}^{\bar{x}_{k}} p(x) d x
$$

If $a_{k}\left(x-\bar{x}_{k}\right)+c_{k} \geq r(x), x \in\left[x_{k-1}, \bar{x}_{k}\right]$, then we define

$$
g_{k}\left(x, c_{k}\right)=a_{k}\left(x-\bar{x}_{k}\right)+c_{k}, x \in\left[x_{k-1}, \bar{x}_{k}\right] .
$$

If $a_{k}\left(x-\bar{x}_{k}\right)+c_{k}<r(x)$ for some $x \in\left[x_{k-1}, \bar{x}_{k}\right]$, we determine $a_{k}$ by the condition

$$
\int_{x_{k-1}}^{x_{k}^{\prime}} r(x) d x+\int_{x_{k}^{\prime}}^{\bar{x}_{k}}\left[a_{k}\left(x-\bar{x}_{k}\right)+c_{k}\right] d x=\int_{x_{k-1}}^{\bar{x}_{k}} p(x) d x,
$$

where $x_{k}^{\prime}$ is such that $a_{k}\left(x_{k}^{\prime}-\bar{x}_{k}\right)+c_{k}=r\left(x_{k}^{\prime}\right)$. We then define

$$
g_{k}\left(x, c_{k}\right)= \begin{cases}r(x) & , \quad x \in\left[x_{k-1}, x_{k}^{\prime}\right], \\ a_{k}\left(x-\bar{x}_{k}\right)+c_{k}, & x \in\left[x_{k}^{\prime}, \bar{x}_{k}\right] .\end{cases}
$$

Likewise, we find $b_{k}$ such that

$$
\int_{\bar{x}_{k}}^{x_{k}}\left[b_{k}\left(x-\bar{x}_{k}\right)+c_{k}\right] d x=\int_{\bar{x}_{k}}^{x_{k}} p(x) d x .
$$


If $b_{k}\left(x-\bar{x}_{k}\right)+c_{k} \geq r(x), x \in\left[\bar{x}_{k}, x_{k}\right]$, we define

$$
h_{k}\left(x, c_{k}\right)=b_{k}\left(x-\bar{x}_{k}\right)+c_{k}, x \in\left[\bar{x}_{k}, x_{k}\right] .
$$

If $b_{k}\left(x-\bar{x}_{k}\right)+c_{k}<r(x)$ for some $x \in\left[\bar{x}_{k}, x_{k}\right]$, we determine $b_{k}$ by the condition

$$
\int_{\bar{x}_{k}}^{x_{k}^{\prime \prime}}\left[b_{k}\left(x-\bar{x}_{k}\right)+c_{k}\right] d x+\int_{x_{k}^{\prime \prime}}^{x_{k}} r(x) d x=\int_{\bar{x}_{k}}^{x_{k}} p(x) d x
$$

where $x_{k}^{\prime \prime}$ satisfies $b_{k}\left(x_{k}^{\prime \prime}-\bar{x}_{k}\right)+c_{k}=r\left(x_{k}^{\prime \prime}\right)$, and define

$$
h_{k}\left(x, c_{k}\right)= \begin{cases}b_{k}\left(x-\bar{x}_{k}\right)+c_{k}, & x \in\left[\bar{x}_{k}, x_{k}^{\prime \prime}\right], \\ r(x), & x \in\left[x_{k}^{\prime \prime}, x_{k}\right] .\end{cases}
$$

We may consider $a_{k}$ and $b_{k}$ to be functions of $c_{k i}$, where $c_{k} \geq p\left(\bar{x}_{k}\right)$. In fact, they are continuous functions for any finite $c_{k}$, since a small change in $c_{k}$ can cause only a small change in either $a_{k}$ or $b_{k}$. We want to show that there is a value of $c_{k}$, say $c_{k}^{\prime}$, such that $a_{k}=b_{k}$, i.e., such that $a_{k}-b_{k}=0$ for $c_{k}=c_{k}^{\prime}$. If $c_{k}=p\left(\bar{x}_{k}\right)$, the convexity of $p(x)$ implies that the corresponding value of $a_{k}-b_{k}$ is non-positive. Furthermore, for $c_{k}$ sufficiently large, the corresponding value of $a_{k}-b_{k}$ is positive. But $a_{k}-b_{k}$ is a continuous function of $c_{k}$ so that the desired value $c_{k}^{\prime}$ exist.

We now let

$$
q_{k}(x)= \begin{cases}g_{k}\left(x, c_{k}^{\prime}\right), & x \in\left[x_{k-1}, \bar{x}_{k}\right], \\ h_{k}\left(x, c_{k}^{\prime}\right), & x \in\left[\bar{x}_{k}, x_{k}\right] .\end{cases}
$$

From (3.18) or (3.19), whichever applies, we have

$$
\int_{x_{k-1}}^{\overline{x_{k}}} q_{k}(x) d x=\int_{x_{k-1}}^{\overline{x_{k}}} p(x) d x .
$$

Hence, the convexity of $p(x)$ and the form of $q_{k}(x)$ imply (by Lemma 1.2) that

$$
\int_{x_{k-1}}^{\bar{x}_{k}} q_{k}(x) u_{n}^{2}(x) d x \geq \int_{x_{k-1}}^{\bar{x}_{k}} p(x) u_{n}^{2}(x) d x .
$$

Similarly, from (3.20) or (3.21) we have

$$
\int_{\bar{x}_{k}}^{x_{k}} q_{k}(x) u_{n}^{2}(x) d x \geq \int_{\bar{x}_{k}}^{x_{k}} p(x) u_{n}^{2}(x) d x .
$$

We are now able to construct the function $q(x)$ by complete induction. To avoid excessive detail, we carry out the proof only up to $n=3$. In $\left(x_{0}, x_{1}\right)$, we set $r(x)=0$, and form $q_{1}(x)$. In $\left(x_{1}, x_{2}\right)$ we also form $q_{2}(x)$ 
with $r(x)=0$. Then, comparing $q_{1}\left(x_{1}\right)$ and $q_{2}\left(x_{1}\right)$, we have the following alternatives:

(i) If $q_{1}\left(x_{1}\right)>q_{2}\left(x_{1}\right)$, we form a new function $q_{2}(x)$ with $r(x)=$ $\max \left[q_{1}(x), 0\right], x \in\left[x_{1}, x_{2}\right]$.

(ii) If $q_{1}\left(x_{1}\right)<q_{2}\left(x_{1}\right)$, we form a new function $q_{1}(x)$ with $r(x)=$ $\max \left[0, q_{2}(x)\right], x \in\left[x_{0}, x_{1}\right]$.

(iii) If $q_{1}\left(x_{1}\right)=q_{2}\left(x_{1}\right)$, we leave $q_{1}(x)$ and $q_{2}(x)$ as they are. Using whichever alternative applies, we define

$$
q^{(1)}(x)= \begin{cases}q_{1}(x), & x \in\left[x_{0}, x_{1}\right], \\ q_{2}(x), & x \in\left[x_{1}, x_{2}\right] .\end{cases}
$$

Now, form $q_{3}(x)$ with $r(x)=0$ for $x \in\left[x_{2}, x_{3}\right]$ and compare $q^{(1)}\left(x_{2}\right)$ and $q_{3}\left(x_{2}\right)$. We have the same alternatives as above, the only difference in procedure being that if $q^{(1)}\left(x_{2}\right)<q_{3}\left(x_{2}\right)$, we must redefine $q^{(1)}(x)$ with $r(x)=\max \left[0, q_{3}(x)\right]$ for $x \in\left[x_{0}, x_{2}\right]$.

It is clear that the induction can be carried out. Furthermore, the resulting function $q(x)$ will be convex, for by the above construction any two adjacent linear segments of the graph of $q(x)$ can only have a common point, such that the corresponding value of $q(x)$ is less than or equal to $p(x)$. Because of this convexity, there is at most one subinterval over which $q(x)=0$. Hence, for each of the points $\bar{x}_{k}, k=1, \cdots, n$, there is at most one vertex of the graph of $q(x)$, except for possibly the one just mentioned. Thus, the graph of $q(x)$ has at most $n+1$ linear segments. That $q(x)$ satisfies the inequality (2.11) follows immediately from (3.22) and (3.23). Hence, our theorem follows as in the proof of Theorem 2.3.

4. Concave densities. In this section, we consider the system (1.5) when $p(x)$ is concave. We prove the following.

THEOREM 4.1. Let $\lambda_{1}[p]$ be the first eigenvalue of a string of unit length with fixed ends whose density is a continuous concave function $p(x)$. Then

$$
\lambda_{1}[p] \int_{0}^{1} p(x) d x \geq \lambda_{0}
$$

where $\lambda_{0}=6.952 \cdots$. The inequality is sharp, equality being attained for a string whose density is the symmetric triangular function

$$
T(x)= \begin{cases}4 x & , \quad x \in[0,1 / 2] \\ 4(1-x), & x \in[1 / 2,1] .\end{cases}
$$

We first assume that $p(x)$ has finite left and right derivatives in the 
closed interval $[0,1]$. We define $f(x)=-p_{-}^{\prime}(x), x \in[0,1]$ where we have set $p_{-}^{\prime}(0)=p_{+}^{\prime}(0)$. We then define

$$
g(x, t)= \begin{cases}x(1-t), & x \in[0, t], \\ (1-x) t, & x \in[t, 1] .\end{cases}
$$

It follows from integration by parts and the fact that

$$
\int_{0}^{1} f(t) d t=p(0)-p(1)
$$

$(p(t)$ is absolutely continuous) that

$$
p(x)=p(0)(1-x)+p(1) x+\int_{0}^{1} g(x, t) d f(t) .
$$

If we set $G(x, t)=2 / t(1-t) g(x, t)$, we get

$$
p(x)=\int_{0}^{1} G(x, t)[t(1-t) / 2] d f(t) .
$$

Here, we have modified $f(t)$ so that the integral includes the terms $p(0)(1-x)$ and $p(1) x$. By Lemma 1.1, we have

$$
\lambda_{1}[p] \int_{0}^{1} p(x) d x \geq \min _{t \in[0,1]} \lambda_{1}[G(x, t)] .
$$

The minimum exists by Lemma 1.3 .

If either the right or left derivative is not finite in $[0,1]$, we consider the system

$$
u^{\prime \prime}+\lambda_{\varepsilon} p u=0, \quad u(\varepsilon)=u(1-\varepsilon)=0,
$$

where $\varepsilon>0$ is small. The above considerations then hold for this system and we have

$$
(1-2 \varepsilon) \lambda_{1}[p]_{\varepsilon} \int_{\varepsilon}^{1-\varepsilon} p(x) d x \geq \min _{t \in[0,1]} \lambda_{1}[G(x, t)] .
$$

Letting $\varepsilon \rightarrow 0$, we have the desired result.

To find the value of $t$ for which $\lambda_{1}[G(x, t)]$ is a minimum, we consider the system

$$
u^{\prime \prime}+\lambda G(x, t) u=0, \quad u(0)=u(1)=0 .
$$

It is convenient to translate the system to the interval $[-1 / 2,1 / 2]$. Thus, we consider

$$
v^{\prime \prime}+\lambda G_{1}(x, t) v=0, \quad v(-1 / 2)=v(1 / 2)=0,
$$

where 


$$
G_{1}(x, t)= \begin{cases}2 \frac{2 x+1}{2 t+1}, & -1 / 2 \leq x \leq t \leq 1 / 2, \\ 2 \frac{1-2 x}{1-2 t}, & -1 / 2 \leq t \leq x \leq 1 / 2 .\end{cases}
$$

We show that $\lambda_{1}\left[G_{1}(x, t)\right]$ is a minimum when $t=0$.

Following Hardy, Littlewood and Polya [6], we define the rearrangement of a non-negative integrable function $g(x), x \in[-1 / 2,1 / 2]$ into a symmetrically decreasing function $\bar{g}(x), x \in[-1 / 2,1 / 2]$. To do this, consider the set $S=\{x \mid g(x) \geq y\}$, where $y$ is some number in the range of $g(x)$. Let $\mu\{S\}$ be the measure of the set $S$. We define the function

$$
m(y)=\mu\{x \mid g(x) \geq y\}
$$

and let

$$
\bar{g}(x)= \begin{cases}m^{-1}(2 x), & x \in[0,1 / 2], \\ \bar{g}(-x), & x \in[-1 / 2,0] .\end{cases}
$$

where $m^{-1}$ denotes the inverse of $m(y)$. In particular, since $m(y)=1-y / 2$ for $g=G_{1}, y \in[0,2]$, we find that this symmetrization transforms $G_{1}(x, t)$ into $G_{1}(x, 0)$. Thus

$$
G_{1}(x, 0)=\bar{G}_{1}(x, t)= \begin{cases}2(1-2 x), & x \in[0,1 / 2], \\ 2(1+2 x), & x \in[-1 / 2,0] .\end{cases}
$$

By a result of Beesack and Schwarz [2], the first eigenvalue of (4.2) is greater than the first eigenvalue of

$$
v^{\prime \prime}+\lambda \bar{G}_{1}(x, t) v=0, \quad v(-1 / 2)=v(1 / 2)=0,
$$

i. e., $\lambda_{1}[G(x, t)] \geq \lambda_{1}[G(x, 1 / 2)]$. Hence, $\lambda_{1}[p] \geq \lambda_{1}[G(x, 1 / 2)]=\lambda_{1}[T(x)]$.

If we solve (4.1) with $t=1 / 2$ [7], we find the eigenvalues to be

$$
\lambda_{n}[G(x, 1 / 2)]=(9 / 2) z_{n}^{2},
$$

where $z_{n}$ is the $n$th positive root of the equation

$$
J_{-2 / 3}(z)=0
$$

and $J_{-2 / 3}$ is the Bessel function of order $-2 / 3$. Numerical calculation gives the result

$$
\lambda_{1}[G(x, 1 / 2)]=6.952 \cdots .
$$

As in the case of monotone and convex densities, we have the following result for the first $n$ eigenvalues of (1.5) when $p(x)$ is concave.

THEOREM 4.2. Let $\lambda_{k}[p], k=1,2, \cdots, n$ be the first $n$ eigenvalues of a string of unit length with fixed ends whose density is a continuous 
concave function $p(x)$. If $\lambda_{k}[G(x, t)], k=1,2, \cdots, n$, are the first $n$ eigenvalues of a string whose density is the triangular function

$$
G(x, t)= \begin{cases}2 \frac{x}{t}, & x \in[0, t], \\ 2\left(\frac{1-x}{1-t}\right), & x \in[t, 1] .\end{cases}
$$

then

$$
\left[\int_{0}^{1} p(x) d x\right]^{-1} \sum_{k=1}^{n} \lambda_{k}^{-1}[p] \sum_{k=1}^{n} \leq \lambda_{k}^{-1}\left[G\left(x, t_{0}\right)\right]
$$

for suitable $t_{0}$.

It is evident that the inequality is sharp. We omit the proof since it contains no new ideas.

For the higher eigenvalues of (1.5), when $p(x)$ is concave, we prove the following.

THEOREM 4.3. Let $\lambda_{n}[p]$ be the $n$th eigenvalue of a string of unit length with fixed ends whose density is a continuous concave function $p(x)$. Then there is a string with the same total mass whose density is a piecewise linear concave function $q(x)$, where the graph of $q(x)$ has at most $n+1$ linear segments and where $q(0)=q(1)=0$, such that

$$
\lambda_{n}[p] \geq \lambda_{n}[q]
$$

where $\lambda_{n}[q]$ is the $n$th eigenvalue of the string with density $q(x)$.

We use the same construction as in the proof of Theorem 3.4. This is possible, for if $p(x)$ is concave, $-p(x)$ is convex.

Let $u_{n}(x)$ be the $n$th eigenfunction of (1.5) when $p(x)$ is concave. As in $\S 2$, we denote the nodal points of $u_{n}(x)$ by $x_{k}, k=0,1,2, \cdots, n$, and the maximum points by $\bar{x}_{k}, k=1, \cdots, n$. Then $-u_{n}^{2}(x)$ has the maximum points $x_{k}, k=0,1, \cdots, x$, and the minimum points $\bar{x}_{k}, k=1$, $2, \cdots, n$. Over each of the intervals $\left(\bar{x}_{k}, \bar{x}_{k+1}\right), k=1, \cdots, n-1$, we way define $-q_{k}\left(x, c_{k}\right)$ where now $-p\left(x_{k}\right) \leq c_{k} \leq 0$, as in Theorem 3.4. As before, there is a value of $c_{k}=c_{k}^{\prime}$ such that $q_{k}\left(x, c_{k}^{\prime}\right)$ is linear at $x=x_{k}$. For the intervals $\left(0, \bar{x}_{1}\right)$ and $\left(\bar{x}_{n}, 1\right)$ we let $c_{0}=0$ and and $c_{n}=0$ so as to define $-q_{0}\left(x, c_{0}\right)$ and $-q_{n}\left(x, c_{n}\right)$. Now using the same induction argument as in Theorem 3.4, we form the functions $-q_{k}\left(x, c_{k}\right)$ and obtain a function $-q(x)$ which is convex and satisfies the inequality

$$
\int_{0}^{1} p(x) u_{n}^{2}(x) d x \leq \int_{0}^{1} q(x) u_{n}^{2} d x .
$$

Here, $r(x)$ is always a linear function and is always negative. The graph of $-q(x)$ consists of at most $n+1$ linear segments, one for every 
point $x_{k}, k=0,1, \cdots, n$, and $q(x)$ is concave. By the argument used in the proof of Theorem 2.3, it then follows that $\lambda_{n}[p] \geq \lambda_{n}[q]$.

5. The general Sturm-Liouville system. We now turn to the SturmLiouville system

$$
\begin{aligned}
& {\left[r(x) u^{\prime}\right]^{\prime}+[\lambda p(x)-q(x)] u=0,} \\
& u^{\prime}(0)-h_{0} u(0)=u^{\prime}(1)+h_{1} u(1)=0,
\end{aligned}
$$

where $p(x)$ and $q(x)$ are non-negative integrable functions, $r(x) \in C^{\prime}$ is positive, and $h_{0} \geq 0, h_{1} \geq 0$. The lowest eigenvalue of this system is given by

$$
\lambda_{1}[p]=\min _{u \in \sigma^{\prime}} \frac{\int_{0}^{1}\left[r(x) u^{\prime 2}+q(x) u^{2}\right] d x}{\int_{0}^{1} p(x) u^{2} d x},
$$

where the functions $u(x)$ satisfy the appropriate bonndary conditions.

It is easy to see that the conclusion of Lemma 1.1 also applies to this differential system, i. e., if $p(x)$ can be expressed in the form (1.8), then

$$
\lambda_{1}[p] \int_{0}^{1} p(x) d x \geq \underset{t \varepsilon[0,1]}{\operatorname{g.l} .1 . b .} \lambda_{1}[K(x, t)],
$$

where $\lambda_{1}[K(x, t)]$ is the first eigenvalue of the system (5.1) with $p(x)$ replaced by $K(x, t)$. Hence, it is possible to generalize Theorems 2.1, 3.1 and 4.1 to the system (5.1). We have

THEOREM 5.1. The densities $p(x)$ minimizing the expression $\lambda_{1}[p] \int_{0}^{1} p(x) d x$, where $\lambda_{1}[p]$ is the lowest eigenvalue of the system (5.1) under the assumptions

(a) $p(x)$ is monotone,

(b) $p(x)$ is convex,

(c) $p(x)$ is concave,

are of the same character as those discussed in Theorems 2.1, 3.1 and 4.1, respectively.

The proof of the theorem presents no new features. It should, however, be noted that the symmetrization argument used in the proof of Theorem 4.1, cannot be applied unless $q(x)$ and $r(x)$ have the symmetric property $q(x)=q(1-x)$ and $r(x)=r(1-x)$. In the general case, all that can be said is that the graph of the extremal density consists of two linear segments passing through $(0,0)$ and $(0,1)$, respectively. It should also be noted that the lack of symmetry in the boundary condition of (5.1) makes it impossible to tell, in general, whether the g.l.b. 
$\lambda_{1}[p]$ for monotone convex $p(x)$ is approached in the case of increasing $p(x)$ or decreasing $p(x)$.

We now consider the system

$$
u^{\prime \prime}+[\lambda p(x)-q(x)] u=0, u(0)=u(1)=0 .
$$

We denote the first eigenvalue of (5.3) by $\lambda_{1}[p, q]$. We have the following lemma.

LEMMA 5.1. Let $q(x)$ be of the form

$$
q(x)=\int_{0}^{1} K(x, t) g(t) d f(t)
$$

where $K(x, t), g(t)$ and $f(t)$ are as defined in Lemma 1.1; then

$$
\lambda_{1}[p, q] \geq \underset{t \in[0,1]}{\mathrm{g} .1 . b} \lambda_{1}[p, Q K(x, t)],
$$

where $\lambda_{1}[p, Q K(x, t)]$ is the lowest eigenvalue of

$$
u^{\prime \prime}+[\lambda p(x)-Q K(x, t)] u=0, \quad u(0)=u(1)=0
$$

and $Q=\int_{0}^{1} q(x) d x$.

By (5.2), we have

$$
\lambda_{1}[p, q]=\min _{u \in O^{\prime}} \frac{\int_{0}^{1}\left\{u^{\prime 2}+\left[\int_{0}^{1} K(x, t) g(t) d f(t)\right] u^{2}\right\} d x}{\int_{0}^{1} p u^{2} d x} .
$$

Hence,

$$
\begin{aligned}
\lambda_{1}[p, q] & \geq \min _{u \in O^{\prime}} \int_{0}^{1} g(t)\left\{\frac{\int_{0}^{1}\left[\left(u^{\prime}\right)^{2} / Q+K(x, t) u^{2}\right] d x}{\int_{0}^{1} p(x) u^{2} d x}\right\} d f(t) \\
& \geq(1 / Q) \int_{0}^{1} g(t) \lambda_{1}[p, Q K(x, t)] d f(t) \\
& \geq(1 / Q) \int_{0}^{1} g(t) d f(t) \underset{t \in[0,1]}{\mathrm{g} . \text { l. b. } \lambda_{1}[p, Q K(x, t)] .}
\end{aligned}
$$

But $Q=\int_{0}^{1} g(t) d f(t)$, so that the conclusion of the lemma follows.

If $q(x)$ is concave, Lemma 5.1 yields the following result.

THEOREM 5.2. The lowest eigenvalue $\lambda_{1}[p, q]$ of (5.3) when $q(x)$ is concave satisfies the inequality

$$
\lambda_{1}[p, q] \geq \min _{t \in[0,1]} \lambda_{1}[p, Q G(x, t)]
$$

where $\lambda_{1}[p, Q G(x, t)]$ is the lowest eigenvalue of (5.3) with $q(x)$ replaced by $Q G(x, t), G(x, t)$ being defined by 


$$
G(x, t)= \begin{cases}2 \frac{x}{t}, & x \in[0, t], \\ 2 \frac{(1-x)}{(1-t)}, & x \in[t, 1] .\end{cases}
$$

We first assume that $q_{+}^{\prime}(0)$ and $q_{-}^{\prime}(1)$ are finite. As in Theorem 4.1, we may express $q(x)$ as

$$
q(x)=\int_{0}^{1} G(x, t)[t(1-t) / 2] d f(t) .
$$

By Lemma 5.1, we have

$$
\lambda_{1}[p, q] \geq \underset{t \in[0,1]}{\operatorname{g} . \text { l. b. }} \lambda_{1}[p, Q G(x, t)] .
$$

If $q_{+}^{\prime}(0)$ or $q_{-}^{\prime}(1)$ are not finite, we consider the system (5.3) with $x$ restricted to the interval $[\varepsilon, 1-\varepsilon]$. Transforming this system to the unit interval, we see that (5.4) applies, so that letting $\varepsilon \rightarrow 0$, we find that (5.4) holds in general for concave $q(x)$.

Since $\lambda_{1}[p, Q G(x, t)]$ is a continuous function of $t \in[0,1]$, there must be a value of $t$ for which the greatest lower bound is attained.

The same procedure can be made to yield corresponding results in the case of monotone and convex densities.

We close this section with some remarks about the system

$$
u^{\prime \prime}+\lambda p(x) u=0, \quad u^{\prime}(0)=u(1)=0,
$$

where $\int_{0}^{1} p d x=1$.

If $p(x)$ is monotone increasing, then $\lambda_{1}$, the lowest eigenvalue of (5.4), satisfies $\lambda_{1} \geq \pi^{2} / 4$.

This follows immediately from Lemma 1.2 if we compare $p(x)$ and $q(x)=1$.

Similarly, if we compare a concave density $p(x)$ and $q(x)=2 x$, we find that $\lambda_{1}[p]$ satisfies the inequality

$$
\lambda_{1} \geq \lambda_{0}
$$

where $\lambda_{0}=6.95 \cdots / 4$ is the lowest eigenvalue of (5.4) with $p(x)=2 x$.

6. The vibrating rod. The eigenvalue problem associated with a rod with clamped ends at $x=a$ and $x=b$ is

$$
y^{i v}-\mu \rho(x) y=0, \quad y(a)=y^{\prime}(a)=y(b)=y^{\prime}(b)=0 .
$$

As in the case of the string, we may transform this system to the unit interval. We have

$$
u^{i v}-\lambda p(x) u=0, \quad u(0)=u^{\prime}(0)=u(1)=u^{\prime}(1)=0,
$$


where $p(x)=(b-a) \rho[(b-a) x+a]$. we note that $\int_{0}^{1} p(x) d x=\int_{a}^{b} p(x) d x$. The eigenvalues $\mu_{n}[\rho], n=1,2, \cdots$, of (6.1) are related to those of (6.2) by the equation

$$
\lambda_{n}[p]=(b-a)^{3} \mu_{n}[\rho], \quad n=1,2, \cdots
$$

The first eigenvalue of (6.2) is equal to the minimum of the Rayleigh quotient

$$
J(p, u)=\frac{\int_{0}^{1}\left(u^{\prime \prime}\right)^{2} d x}{\int_{0}^{1} p(x) u^{2} d x}
$$

where $u(x)$ ranges over all functions $u \in C^{2}$ such that $u(0)=u^{\prime}(0)=0$ and $u(1)=u^{\prime}(1)=0$.

The following results correspond to Theorems 2.1, 3.1 and 4.1 for the string.

TheOREm 6.1. Let $\lambda_{1}[p]$ be the lowest eigenvalue of a rod of unit length with clamped ends. From the assumptions that

(a) $p(x)$ is monotone,

(b) $p(x)$ is convex,

(c) $p(x)$ is concave,

we have

$$
\lambda_{1}[p] \int_{0}^{1} p(x) d x \geq \lambda_{1}\left[K\left(x, t_{0}\right)\right]
$$

where

$$
\begin{aligned}
& \left(a^{\prime}\right) \quad K\left(x, t_{0}\right)= \begin{cases}0, & x \in\left[0, t_{0}\right], \\
\frac{1}{1-t_{0}}, & x \in\left(t_{0}, 1\right],\end{cases} \\
& \left(b^{\prime}\right) \quad K\left(x, t_{0}\right)= \begin{cases}0, & x \in\left[0, t_{0}\right], \\
\frac{2\left(x-t_{0}\right)}{\left(1-t_{0}\right)^{2}}, & x \in\left(t_{0}, 1\right],\end{cases} \\
& \left(c^{\prime}\right) K\left(x, t_{0}\right)= \begin{cases}2 \frac{x}{t_{0}}, & x \in\left[0, t_{0}\right], \\
2 \frac{1-x}{\left(1-t_{0}\right)}, & x \in\left[t_{0}, 1\right]\end{cases}
\end{aligned}
$$

respectively, for suitable values of $t_{0}$.

There is nothing new involved in the proof over that of the corresponding theorems for the string. In fact, we need only replace the 
Rayleigh quotient of (1.5) by (6.3) and the respective proofs for the corresponding string problem apply.

In the case of concave $p(x)$, it can be shown that $\lambda_{1}[p]$ takes its smallest value for $t_{0}=1 / 2$, i. e., we have the following result.

THEOREM 6.2. If $\lambda_{1}[p]$ is the lowest eigenvalue of a rod with clamped ends whose density function is a positive concave function $p(x)$, then

$$
\lambda_{1}[p] \int_{0}^{1} p d x \geq \lambda_{1}\left[p_{0}\right]
$$

where

$$
p_{0}(x)= \begin{cases}4 x & , \quad x \in[0,1 / 2] \\ 4(1-x), & x \in[1 / 2,1] .\end{cases}
$$

The proof will be based on the following result of Beesack [1].

THEOREM 6.3. Let $p(x)$ be continuous and non-negative for $x \in$ $[-1 / 2,1 / 2]$ and let $\bar{p}(x)$ be the rearrangement of $p(x)$ into symmetrically decreasing order. Then the first eigenvalues of the system

$$
u^{i v}-\lambda p(x)=0, \quad u(-1 / 2)=u^{\prime}(-1 / 2)=u(1 / 2)=u^{\prime}(1 / 2)=0,
$$

and

(6.5) $\quad v^{i v}-\mu \bar{p}(x) v=0, \quad v(-1 / 2)=v^{\prime}(-1 / 2)=v(1 / 2)=v^{\prime}(1 / 2)=0$.

satisfy the condition

$$
\mu_{1}[\bar{p}] \leq \lambda_{1}[p] .
$$

The rearrangement of $p(x)$ into symmetrically decreasing order is defined as above in Theorem 4.1.

The proof of Theorem 6.2 follows immediately from Theorems 6.1 and 6.3 , since the symmetrization of

$$
K(x, t)= \begin{cases}2 \frac{x}{t}, & x \in[0, t], \\ 2 \frac{1-x}{1-t}, & x \in[t, 1],\end{cases}
$$

is $K(x, 1 / 2)$.

Theorem 6.3 also leads to a result corresponding to that of Krein for a string with a bounded density function.

THEOREM 6.4. Let $p(x)$ satisfy the condition $0 \leq p(x) \leq H<\infty$, $x \in[0,1]$. Then the lowest eigenvalue $\lambda_{1}[p]$ of a rod with clamped ends 
and density $p(x)$ satisfies the inequality

$$
\lambda_{1}[p] \int_{0}^{1} p(x) d x \geq \lambda_{0},
$$

where $\lambda_{0}$ is the lowest eigenvalue of the rod with density

$$
p_{0}(x)=\left\{\begin{array}{l}
0, \quad x \in\left[0,1 / 2-\frac{M}{2 H}\right), \\
H, x \in\left[1 / 2-\frac{M}{2 H}, 1 / 2+\frac{M}{2 H}\right], \\
0, x \in\left(1 / 2+\frac{M}{2 H}, 1\right],
\end{array}\right.
$$

and where $M=\int_{0}^{1} p(x) d x$.

Let $y_{1}(x)$ be the first eigenfunction of a rod with clamped ends and density $\bar{p}(x)$, (the function resulting from symmetrization of $p(x)$ about $x=1 / 2)$. Then it is clear that $p_{0}(x), \bar{p}(x)$ and $y_{1}^{2}(x)$ satisfy the hypothesis of Lemma 1.2 over the interval $[0,1 / 2]$ so that

$$
\int_{0}^{1 / 2} \bar{p}(x) y_{1}^{2}(x) d x \leq \int_{0}^{1 / 2} p_{0}(x) y_{1}^{2}(x) d x
$$

By symmetry, we have

$$
\int_{1 / 2}^{1} \bar{p}(x) y_{1}^{2}(x) d x \leq \int_{1 / 2}^{1} p_{0}(x) y_{1}^{2}(x) d x
$$

Adding these two inequalities, we find

$$
\lambda_{1}[\bar{p}]=\frac{\int_{0}^{1}\left(y_{1}^{\prime \prime}\right)^{2} d x}{\int_{0}^{1} \bar{p}(x) y_{1}^{2} d x} \geq \frac{\int_{0}^{1}\left(y_{1}^{\prime \prime}\right)^{2} d x}{\int_{0}^{1} p_{0}(x) y_{1}^{2} d x} \geq \lambda_{0} .
$$

Hence, by Theorem 6.2 we have $\lambda_{1}[p] \geq \lambda_{0}$.

We close this section with the remark that corresponding versions of Theorem 6.1 hold if we replace the boundary conditions (6.2) by any of the other boundary conditions used in the theory of the vibrating rod.

7. The vibrating membrane. We consider a vibrating membrant covering a simply connected domain $D$ whose boundary is a Jordan curve $C$. Let $p(x, y)$ be the density of the membrane. We assume that $p(x, y$. is measurable and that

$$
0 \leq p(x, y) \leq H<\infty, \quad(x, y) \in D,
$$


The eigenfunctions and the eigenvalues of this membrane, with the boundary fixed at $(x, y) \in C$, are determined by the integral equation [14]

$$
u(x, y)=\lambda \iint_{D} G(x, y, \xi, \eta) p(\xi, \eta) u(\xi, \eta) d \xi d \eta,
$$

where $G(x, y, \xi, \eta)$ is the Green's function of the domain $D$. We denote the first eigenvalue by $\lambda_{1}[p]$.

We define $M$ and $R$ by the relations

$$
M=\iint_{D} p(x, y) d x d y, \quad \pi R^{2}=\iint_{D} d x d y
$$

and let $D^{*}$ be the circle $x^{2}+y^{2} \leq R^{2}$. In this section, we prove the following two theorems concerning $\lambda_{1}[p]$.

THEOREM 7.1. The minimum of $\lambda_{1}[p]$, subject to the restrictions (7.1) and (7.3), is given by a membrane covering $D^{*}$ with density

$$
\bar{p}_{0}(x, y)= \begin{cases}H, & x^{2}+y^{2} \leq \rho^{2} \\ 0, & \rho^{2} \leq x^{2}+y^{2} \leq R^{2}\end{cases}
$$

where $\rho$ is defined by $\pi \rho^{2} H=M$.

Let $D$ be a convex domain. $p(x, y)$ is concave in $D$ if, for $\left(x_{1}, y_{1}\right) \in D$ and $\left(x_{2}, y_{2}\right) \in D$, we have

$$
p\left(\frac{x_{1}+x_{2}}{2}, \frac{y_{1}+y_{2}}{2}\right) \leq(1 / 2)\left[p\left(x_{1}, y_{1}\right)+p\left(x_{2}, y_{2}\right)\right] \text {. }
$$

For a concave density function, we have the following result.

THEOREM 7.2. Let $\lambda_{1}[p]$ be the lowest eigenvalue of a membrane, with fixed edges, covering a convex domain $D$, whose density function is concave. Then

$$
\lambda_{1}[p] \iint_{D} p(x, y) d x d y \geq \pi \lambda_{0},
$$

where $\lambda_{0}=3.26 \cdots$. The inequality is sharp and equality is attained for a circular membrane of radius $R$ and density

$$
p_{0}(x, y)=p_{0}(r)=\frac{3 M}{\pi R^{3}}(R-r), \quad 0<r \leq R,
$$

where $r^{2}=x^{2}+y^{2}$.

Krein [8] has conjectured the result of Theorem 7.1 for the case where $D=D^{*}$. The numerical value of the minimum is given by the least positive root of 


$$
J_{0}(\sqrt{\lambda H} \rho)-\rho J_{0}^{\prime}(\sqrt{\lambda H} \rho) \sqrt{\lambda H} \ln \rho / R=0 .
$$

To prove these theorems, we use the extremal characterization of $\lambda_{1}[p]$, i. e., the first eigenvalue $\lambda_{1}[p]$ of (7.2) is given by

$$
\lambda_{1}[p]=\underset{u}{\text { g. l. b. } J(p, u),}
$$

where $J(p, u)$ is the Rayleigh quotient

$$
J(p, u)=\frac{\iint_{D}|\operatorname{grad} u(x, y)|^{2} d x d y}{\iint_{D} p(x, y) u^{2}(x, y) d x d y}
$$

and where the greatest lower bound is taken over all continuous functions with piecewise continuous first derivatives, such that $u=0$ on $C$.

As the following lemma shows, the same result is obtained if $u$ is made subject to additional restrictions.

Lemma 7.1. The first eigenvalue $\lambda_{1}[p]$ of (7.2) is given by

$$
\lambda_{1}[p]=\text { g. l. b. } J(p, u)
$$

where $J(p, u)$, is the Rayleigh quotient (7.7) and where the greatest lower bound is taken over all analytic functions $u(x, y)$ with $u=0$ on $C$.

Since $p(x, y)$ is a measurable function, $\sqrt{p(x, y)}$ is measurable. Hence, there is a polynomial, $Q(x, y)$, such that for arbitrary numbers $\delta, \eta>0$, we have

$$
|\sqrt{p(x, y)}-Q(x, y)|<\delta
$$

except on a set of measure less than $\eta$. Furthermore, $Q(x, y)$ may be chosen such that $q(x, y)=Q^{2}(x, y)$ is non-negative and is less than $H$.

We consider the membrane over the domain $D$ with density $q(x, y)$. The eigenfunctions and eigenvalues will be determined by (7.2) with $p(x, y)$ replaced by $q(x, y)$. In particular, we denote the first eigenvalue by $\lambda_{1}[q]$. Since $q(x, y)$ is an analytic function in $D$, it is well known that

$$
\lambda_{1}[q]=\min _{u \in \sigma^{\infty}} J(q, u),
$$

where the minimum is taken over all the indicated functions for which $u \equiv 0$ on $C$. It is also well known that the eigenvalues of (7.2) are continuous functionals of $p(x, y)$. More precisely, for any $\varepsilon>0$, there is a $\delta_{1}>0$ such that

(7.11) $\iint_{D} \iint_{D} G^{2}(x, y, \xi, \eta)\left[\sqrt{p(x, y) p(\xi, \eta)}-\sqrt{q(x, y) q(\xi, \eta)]^{2}} d x d y d \xi d \eta<\delta_{1}\right.$ 
implies

$$
\left|\frac{1}{\lambda_{1}[p]}-\frac{1}{\lambda_{1}(q)}\right|<\varepsilon .
$$

It is easy to see that $\delta$ and $\eta$ may be chosen so that (7.9) implies (7.11) and hence, also (7.12).

For any analytic function $u(x, y)$ such that $u=0$ on $C$, we now show that there are values of $\delta$ and $\eta$ such that (7.9) implies

$$
\left|[J(p, u)]^{-1}-[J(q, u)]^{-1}\right|<\varepsilon
$$

where $\varepsilon>0$ is arbitrary and $\delta$ and $\eta$ are independent of $u(x, y)$.

We have

$$
A=\left|[J(p, u)]^{-1}-[J(q, u)]^{-1}\right| \leq[J(|p-q|, u)]^{-1} .
$$

Now $|p-q|=|\sqrt{p}+\sqrt{q}||\sqrt{p}-\sqrt{q}| \leq 2 \sqrt{H \delta}$, except on a set $S$ of measure less than $\eta$. Hence,

$$
A \leq 2 \sqrt{H} \delta[J(1, u)]^{-1}+H \eta \frac{\max _{(x, y) \in D} u(x, y)}{\iint_{D}|\operatorname{grad} u|^{2} d x d y} .
$$

By Rayleigh's theorem on the first eigenvalue of a homogeneous membrane [11], $J(1, u) \geq j_{0}^{2} / R^{2}$, where $j_{0}$ is the least positive zero of the Bessel function $J_{0}(x)$ and $R$ is defined by (7.3). Furthermore, if we let $u_{m}=\max _{(x, y) \in D} u(x, y)$, then

$$
\iint_{D}\left|\operatorname{grad} \frac{u}{u_{m}}\right|^{2} d x d y \geq 4 \pi c,
$$

where $c$ is the capacity of an infinite circular cylinder of radius $R$ with zero potential on the surface of the cylinder and potential one on the axis of the cylinder [11]. Hence, we have

$$
A \leq 2 \sqrt{H} \frac{R^{2}}{j_{0}^{2}} \sigma+\frac{H}{4 \pi c} \eta,
$$

so that (7.13) follows.

Let $u_{1}(x, y)$ be the first eigenfunction corresponding to $\lambda_{1}[q]$. We may choose $\delta$ and $\eta$ so that (7.12) and (7.13) hold simultaneously. Hence, we have

$$
\begin{aligned}
\left|\lambda_{1}^{-1}[p]-\left[J\left(p, u_{1}\right)\right]\right| \leq & \left|\lambda_{1}^{-1}[p]-\lambda_{1}^{-1}[q]\right| \\
& +\left|\left[J\left(q, u_{1}\right)\right]-\left[J\left(p, u_{1}\right)\right]\right| \leq 2 \varepsilon,
\end{aligned}
$$

for some function $u_{1}(x, y)$ which is analytic in $D$.

By (7.13) we have, for any analytic function $u(x, y)$ that 


$$
[J(p, u)]^{-1}<[J(q, u)]^{-1}+\varepsilon .
$$

(7.10) and (7.12) then give

$$
[J(p, u)]^{-1}<\lambda_{1}^{-1}[q]+\varepsilon<\lambda_{1}^{-1}[p] 2 \varepsilon .
$$

Since $\varepsilon$ is small, we finally conclude that $\lambda_{1}[p] \leq J(p, u)$. This with (7.14) gives (7.7).

We now introduce the symmetrization of $p(x, y)$ with respect to a line perpendicular to the $(x, y)$-plane, i. e., Schwarz symmetrization [11]. We may define it by considering the function

$$
a(\rho)=\mu\{(x, y), p(x, y) \geq \rho\}
$$

where $\mu$ denotes the measure of the set indicated and where $\rho$ is some number between 0 and $H$. Then the symmetrization of $p(x, y)$ is

$$
\bar{p}(x, y)=\bar{p}(r)=a^{-1}\left(\pi r^{2}\right), \quad r \in[0, R]=D^{*},
$$

where $r^{2}=x^{2}+y^{2}$.

We now prove the following.

LEMmA 7.2. The lowest eigenvalue $\lambda_{1}[p]$ of (7.2) is bounded below by the lowest eigenvalue $\lambda_{1}[\bar{p}]$ of the membrane with fixed boundary over $D^{*}$ and density $\bar{p}(x, y)$.

B. Schwarz [15] has shown that when $p(x, y) \in C^{\prime}$,

$$
\lambda_{1}[p] \geq \underset{u \in O^{\prime}}{\operatorname{g} . \log } . J[\bar{p}, u],
$$

where now the Rayleigh quotient is defined over $D^{*}$. By Lemma 7.1, it follows that $\lambda_{1}[p] \geq \lambda_{1}[\bar{p}]$. The proof of Lemma 7.2 differs only in detail from the proof of the result of Schwarz.

By Lemma 7.1, there is an analytic function $u(x, y)$ such that

$$
\lambda_{1}[p]+\varepsilon \geq J(p, u)
$$

where $\varepsilon>0$ is arbitrary. Let $\bar{u}(x, y)=\bar{u}(r), r \in D^{*}$ be the above symmetrization of $u(x, y)$. Schwarz shows that such a symmetrization of an analytic function gives a function with piecewise continuous first derivatives and it is further known [11] that

$$
\iint_{D}|\operatorname{grad} u|^{2} d x d y \geq \iint_{D^{*}}|\operatorname{grad} \bar{u}|^{2} r d r d \theta .
$$

We also known [6], [11] that

$$
\iint_{D} p(x, y) u^{2}(x, y) d x d y \leq \iint_{D^{*}} \bar{p}(r) \bar{u}^{2}(r) r d r d \theta .
$$

Hence, we have from (7.16) and Lemma 7.1 that 


$$
\lambda_{1}[p]+\varepsilon \geq J(\bar{p}, \bar{u}) \geq \lambda_{1}[\bar{p}] .
$$

But $\varepsilon$ is arbitrary so that Lemma 7.2 follows.

We now prove Theorem 7.1. By Lemma 7.1, there is a symmetric and analytic function $\bar{u}(x, y)=\bar{u}(r),(x, y) \in D^{*}$, such that for arbitrary $\varepsilon>0$,

$$
\lambda_{1}[\bar{p}]+\varepsilon \geq J(\bar{p}, \bar{u}) .
$$

$\bar{u}(r)$ may be chosen such that it is the first eigenfunction of a membrane with a symmetric, analytic density $\bar{q}(x, y)=\bar{q}(r),(x, y) \in D^{*}$. In this, case, the integral equation which gives $\bar{u}(r)$ is equivalent to the partial differential equation of this membrane. It is easily seen that $\bar{u}(r)$ must have its only maximum at $r=0$. We now compare the integrals

$$
\iint_{D^{*}} \bar{p}(r) \bar{u}(r) r d r d \theta
$$

and

$$
\iint_{D^{*}} \bar{p}_{0}(r) \bar{u}(r) r d r d \theta
$$

where $\bar{p}_{0}(r)=\bar{p}_{0}(x, y)$ is defined by (7.4). From the definition of $\bar{p}(r)$ we have $0 \leq \bar{p}(r) \leq H, 0<r \leq R$. Hence, $\bar{p}(r) r$ and $\bar{p}_{0}(r) r$ satisfy the same relationship as $p(x)$ and $q(x)$ of Lemma 1.2. It then follows that

$$
\lambda_{1}[\bar{p}]+\varepsilon \geq J\left(\bar{p}_{0} \bar{u}\right) .
$$

By Lemma 7.1, we have $\lambda_{1}[\bar{p}] \geq \lambda_{1}\left[\bar{p}_{0}\right]$, since $\epsilon$ is arbitrary. In view of Lemma 7.2, this proves Theorem 7.1.

To prove Theorem 7.2 , we again consider $\bar{p}(x, y)=\bar{p}(r),(x, y) \in D^{*}$. This function is obtained by Schwarz symmetrization from $p(x, y),(x, y) \in D$, where $D$ is a convex domain. We show that if $p(x, y)$ is concave, then so is $\bar{p}(x, y)$.

Consider the three dimensional set

$$
S=\{(x, y, z) \mid(x, y) \in D, \quad 0 \leq z \leq p(x, y)\} .
$$

This set is convex and Steiner symmetrization, i. e., symmetrization with respect to a plane, preserves convexity [3]. Furthermore, $\bar{p}(x, y)$ may be obtained by an infinite number of Steiner symmetrizations with respect to planes through the origin which are perpendicular to $(x, y)$-plane [3], [11]. This symmetrization of $S$ gives

$$
\bar{S}=\{(x, y, z) \mid(x, y) \in D, \quad 0 \leq z \leq \bar{p}(x, y)\} .
$$

Clearly, $\bar{p}(x, y)$ will then be a concave function.

As in the proof of Theorem 7.1, there is an analytic function 
$\bar{u}(x, y)=\bar{u}(r)$ whose only extremal value is the maximum at $r=0$ such that $\lambda_{1}[\bar{p}]+\varepsilon \geq J(\bar{p}, \bar{u})$. Since $\bar{u}(r)$ is concave, $\bar{p}_{0}(r)$ and $\bar{p}(r)$ satisfy the relation $\bar{p}_{0}(r) \geq \bar{p}(r)$ for $r \in\left(0, r_{0}\right)$ where $r_{0} \in(0, R)$ and $\bar{p}_{0}(r) \leq \bar{p}(r)$ for $r \in\left(r_{0}, R\right)$. Hence, $r \bar{p}_{0}(r)$ and $r \bar{p}(r)$ are related in the same way as $p(x)$ and $q(x)$ in Lemma 1.2. As in Theorem 7.1, we have $\lambda_{1}[\bar{p}] \geq \lambda_{1}\left[\bar{p}_{0}\right]$. By Lemma 7.2, Theorem 7.2 then follows.

Using well-known techniques for the computation of eigenvalues [4], we find that the lowest eigenvalue $\lambda_{1}[p]$ of $(7.2)$, where $p(x, y)$ is concave, satisfies the relation

$$
\iint_{D} p(x, y) d x d y \cdot \lambda_{1}[p] \geq(3.26 \cdots) \pi \text {. }
$$

$Z$. Nehari has shown [10] that if $p(x, y)$ is superharmonic, then $\lambda_{1}[p]$ satisfies

$$
\iint_{D} p(x, y) d x d y \lambda_{1}[p] \leq \pi j_{0}^{2}
$$

where $j_{0}$ is the least positive zero of $J_{0}(x)$, the Bessel function of order zero. But a concave function is superharmonic [12] so that the bound also applies in our problem. Thus, if $p(x, y)$ is concave in a convex domain,

$$
3.26 \pi \leq \iint_{D} p(x, y) d x d y \lambda_{1}[p] \leq \pi j_{0}^{2}
$$

\section{REFERENCES}

1. P. R. Beesack, Isoperimetric inequalities for the nonhomogeneous clamped rod and plate, J. Math. Mech. 8, pp. 471-482.

2. P. R. Beesack and B. Schwarz, On the zeros of solutions of second order linear differential equations, Canadian J. Math., 8 (1956) 504-515.

3. W. Blaschke, Kreis und Kugel, Chelsea Publishing Co., 1949.

4. L. Collatz, Eigenwertprobleme und ihre Numerische Behandlung, Chelsea Publishing Co., 1948.

5. R. Courant and D. Hilbert, Methods of Mathematical Physics, Interscience Publishers, 1953.

6. G. H. Hardy, J. E. Littlewood, and G. Polya, Inequalities, Cambridge University Press, 1952.

7. E. Jahnke and Emde, Tables of Functions, Dover, 1945.

8. M. G. Krein, On certain problems on the maximum and minimum of characteristic values and on the Lyapunov zones of stability, Amer. Math. Soc. Trans., Series 2, 1, (1955), 163-187.

9. National Bureau of Standards, Tables of Bessel Functions of Fractional Order, Vol. I, Columbia University Press, 1948.

10. Z. Nehari, On the principal frequency of a membrane, Pacific J. Math. 8, (1958) 285293.

11. G. Polya and G. Szego, Isoperimetric Inequalities in Mathematical Physics, Princeton University Press, 1951. 
12. T. Rado, Subharmonic Functions, Chelsea Publishing Co., 1949.

13. E. C. Titchmarsh, The Theory of Functions, Oxford University Press, 1939.

14. F. G. Tricomi Integral Equations, Interscience Publishers, 1957.

15. B. Schwarz, Bounds for the principal frequency of the non-homogeneous membrane, Pacific J. Math. 7, (1957), 1653-1676.

CARNEGIE INSTITUTE OF TEChNOLOGY 


\section{PACIFIC JOURNAL OF MATHEMATICS}

\section{EDITORS}

David Gilbarg

Stanford University

Stanford, California

F. H. Brownell

University of Washington

Seattle 5, Washington
A. L. Whiteman

University of Southern Californıa Los Angeles 7, California

L. J. PaIge

University of California

Los Angeles 24, California

\section{ASSOCIATE EDITORS}
E. F. BECKENBACH
T. M. CHERRY
D. DERRY

E. HEWITT
A. HORN
L. NACHBIN
M. OHTSUKA

H. L. ROYDEN

M. M. SCHIFFER
E. SPANIER

E. G. STRAUS

F. WOLF

\section{SUPPORTING INSTITUTIONS}

\author{
UNIVERSITY OF BRITISH COLUMBIA \\ CALIFORNIA INSTITUTE OF TECHNOLOGY \\ UNIVERSITY OF CALIFORNIA \\ MONTANA STATE UNIVERSITY \\ UNIVERSITY OF NEVADA \\ NEW MEXICO STATE UNIVERSITY \\ OREGON STATE COLLEGE \\ UNIVERSITY OF OREGON \\ OSAKA UNIVERSITY \\ UNIVERSITY OF SOUTHERN CALIFORNIA
}

\author{
STANFORD UNIVERSITY \\ UNIVERSITY OF TOKYO \\ UNIVERSITY OF UTAH \\ WASHINGTON STATE COLLEGE \\ UNIVERSITY OF WASHINGTON \\ AMERICAN MATHEMATICAL SOCIETY \\ CALIFORNIA RESEARCH CORPORATION \\ HUGHES AIRCRAFT COMPANY \\ SPACE TECHNOLOGY LABORATORIES \\ NAVAL ORDNANCE TEST STATION
}

Mathematical papers intended for publication in the Pacific Journal of Mathematics should be typewritten (double spaced), and the author should keep a complete copy. Manuscripts may be sent to any one of the four editors. All other communications to the editors should be addressed to the managing editor, L. J. Paige at the University of California, Los Angeles 24, California.

50 reprints per author of each article are furnished free of charge; additional copies may be obtained at cost in multiples of 50 .

The Pacific Journal of Mathematics is published quarterly, in March, June, September, and December. The price per volume (4 numbers) is $\$ 12.00$; single issues, $\$ 3.50$. Back numbers are available. Special price to individual faculty members of supporting institutions and to individual members of the American Mathematical Society: $\$ 4.00$ per volume; single issues, $\$ 1.25$.

Subscriptions, orders for back numbers, and changes of address should be sent to Pacific Journal of Mathematics, 2120 Oxford Street, Berkeley 4, California.

Printed at Kokusai Bunken Insatsusha (International Academic Printing Co., Ltd.), No. 6, 2-chome, Fujimi-cho, Chiyoda-ku, Tokyo, Japan.

PUBLISHED BY PACIFIC JOURNAL OF MATHEMATICS, A NON-PROFIT CORPORATION

The Supporting Institutions listed above contribute to the cost of publication of this Journal, but they are not owners or publishers and have no responsibility for its content or policies. 


\section{Pacific Journal of Mathematics}

\section{Vol. 10, No. $2 \quad$ October, 1960}

Maynard G. Arsove, The Paley-Wiener theorem in metric linear spaces ........

Robert (Yisrael) John Aumann, Acceptable points in games of perfect

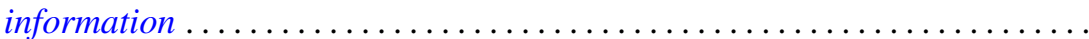

A. V. Balakrishnan, Fractional powers of closed operators and the semigroups generated by them ... . . . . . . . . . . . . . . . . . . . . . . . . . . . . 419

Dallas O. Banks, Bounds for the eigenvalues of some vibrating systems . . . . . 439

Billy Joe Boyer, On the summability of derived Fourier series . . . . . . . . . . . 475

Robert Breusch, An elementary proof of the prime number theorem with

remainder term ...................................

Edward David Callender, Jr., Hölder continuity of $n$-dimensional

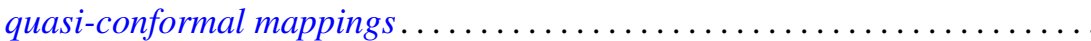

L. Carlitz, Note on Alder's polynomials ......................... 517

P. H. Doyle, III, Unions of cell pairs in $E^{3} \ldots \ldots \ldots \ldots \ldots \ldots \ldots \ldots \ldots \ldots \ldots \ldots . \ldots 21$

James Eells, Jr., A class of smooth bundles over a manifold . . . . . . . . . . . . 525

Shaul Foguel, Computations of the multiplicity function . . . . . . . . . . . . . . 539

James G. Glimm and Richard Vincent Kadison, Unitary operators in

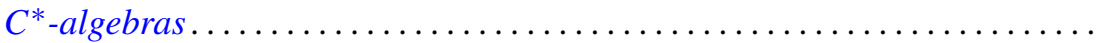

Hugh Gordon, Measure defined by abstract $L_{p}$ spaces . . . . . . . . . . . 557

Robert Clarke James, Separable conjugate spaces ....................

William Elliott Jenner, On non-associative algebras associated with bilinear forms

Harold H. Johnson, Terminating prolongation procedures

John W. Milnor and Edwin Spanier, Two remarks on fiber homotopy type .

Donald Alan Norton, A note on associativity . .

Ronald John Nunke, On the extensions of a torsion module.

Joseph J. Rotman, Mixed modules over valuations rings . . . . .

A. Sade, Théorie des systèmes demosiens de groupoï des . .

Wolfgang M. Schmidt, On normal numbers . .

661

Berthold Schweizer, Abe Sklar and Edward Oakley Thorp, The metrization of

statistical metric spaces

John P. Shanahan, On uniqueness questions for hyperbolic differential

equations

A. H. Stone, Sequences of coverings

Edward Oakley Thorp, Projections onto the subspace of compact operators

L. Bruce Treybig, Concerning certain locally peripherally separable spaces

Milo Wesley Weaver, On the commutativity of a correspondence and a

permutation

David Van Vranken Wend, On the zeros of solutions of some linear complex

differential equations. 